\title{
Adverse selection in the annuity market with sequential and simultaneous insurance demand
}

\author{
Johann K. Brunner · Susanne Pech
}

Revised: 6 October 2006

(C) Springer Science + Business Media, LLC 2006

\begin{abstract}
This paper investigates the effect of adverse selection on the private annuity market in a model with two periods of retirement and two types of individuals, who differ in their life expectancy. In order to introduce the existence of time-limited pension insurance, we consider a model where for each period of retirement separate contracts can be purchased. Demand for the two periods can be decided sequentially or simultaneously. We show that only a situation where all risk types choose sequential contracts is an equilibrium and that this outcome is favourable for the long-living, but is unfavourable for the short-living individuals.
\end{abstract}

Keywords Annuity market · Adverse selection · Uncertain lifetime · Equilibrium

JEL Classification D82 - D91 · G22

\section{Introduction}

Social security systems, which in many industrialised countries are organised according to the pay-as-you-go method, are threatened by the ageing of the population due to a decrease in fertility and an increase in life expectancy. This problem is recognised by academics as well as by politicians, and several possible measures to maintain the financial stability of the system are suggested. One of these measures is a reduction of the pension payments, and it seems in fact unavoidable that it will be implemented to some degree. If this is the case, then a natural strategy for the individuals is to raise private provision for retirement, in particular by an increased purchase of life

J. K. Brunner $\cdot$ S. Pech $(\bowtie)$

Department of Economics, University of Linz, Altenberger Straße 69, A-4040 Linz, Austria

e-mail: susanne.pech@jku.at

J. K. Brunner

e-mail: johann.brunner@jku.at 
annuities. As governments want to prevent old-age poverty, they tend to encourage private pension insurance through tax incentives. ${ }^{1}$

However, there are concerns that the market for annuities does not offer a suitable supplement to the public pension system. One obvious argument is that it cannot incorporate redistribution, as the public system does for several reasons. Another argument concentrates on the phenomenon of adverse selection, which is a common problem that affects the efficient working of insurance markets. The present paper studies this problem in the context of specifically designed contracts for old-age insurance.

Generally, adverse selection occurs with asymmetric information, that is, when the insurer has less information than the individual as to the probability that the insured event occurs. In case of annuities, this means that companies have less information on life expectancy of an annuitant than the individual herself. As a consequence, returns from annuities cannot reflect individual life expectancy but only overall life expectancy, which in turn will induce high-risk individuals (that is, the long-living) to buy more annuities than low-risk individuals. This is the standard observation, discussed in various contributions to the literature [see, e.g., Pauly, 1974; Eckstein et al., 1985; Abel, 1986; Mitchell et al., 1999; Walliser, 2000].

However, there is a further consequence of the adverse-selection problem, namely that the time structure of the benefits matters. Individuals with low life expectancy put less weight on pension payouts in later periods than individuals with high life expectancy. This aspect is neglected in the usual overlapping-generations model with one working period and one period of retirement. But in reality the time of retirement must not be seen as a single, homogeneous period, for which provision can be made through a once-and-for-all contract only, with a fixed and constant (in nominal or real terms) payout. Planning individuals, being aware of some estimate of their life expectancy, will attempt to make provision in accordance with this estimate, which means that they want to use more differentiated instruments. In practice, they can buy an insurance contract with payouts increasing or decreasing over time, or they can buy a time-limited contract for the earlier phase of retirement and then use another instrument to provide for the rest of their lifetime. ${ }^{2}$

In order to analyse the consequences on the functioning of the annuity market of the fact that the time structure of the payouts matters, one has to extend the standard model by assuming that retirement consists of more periods and that provision can be made separately for each of them. Brunner and Pech [2005] introduce a model with one working period and two periods of retirement, where two groups of individuals with differing life expectancy buy an annuity contract which runs for the whole time

\footnotetext{
1 Tax incentives are granted in many industrialised countries, e.g. in Great Britain, U.S.A, Canada and Sweden. Moreover, the recent reform of social security in Germany aims at cutting public pensions and inducing individuals, by granting a tax release, to contribute four percent of income to private old-age insurance. Similarly, in Austria contributions to private old-age insurance are subsidised by a premium.

${ }^{2}$ Poterba [1997] emphasizes the importance of the wide range of different annuity products for the growth of the U.S. annuity market. He provides a typology of individual annuities with respect to the terms under which accumulated capital is dispersed during the liquidation phase. In particular, he distinguishes between two broad classes of individual annuities, that are deferred and immediate annuities, depending on whether there is a waiting period between the premium payment and the beginning of the annuity payouts or not. The role of annuity contracts with escalating payouts in the U.K. annuity market is studied by Finkelstein and Poterba [2002].
} 
of retirement, but with payouts possibly varying over time. ${ }^{3}$ It is shown that in this framework a separating equilibrium may but need not exist. ${ }^{4}$

In the present contribution we consider a similar model, but with different types of contracts. We also assume that individuals live for one working period and for at most two periods of retirement, but now contracts run for one period only; for the second period, a new contract has to be bought. By this formulation we take account of the fact that in reality term-insured pension contracts exist, which provide payouts only for a limited time, given that the individual is alive. For the rest some other form of provision must be made. ${ }^{5}$

The important issue which we address is that individuals can choose between two strategies to provide for the second period of retirement: simultaneously, that is, individuals buy an additional contract already in the working period, or sequentially, that is, only those individuals who have survived to the first period of retirement, purchase an additional contract on the spot market. We show that in our model individuals in general chose only one of these alternatives, depending on the prices. However, in a first-best equilibrium, prices that then correspond to the individual life expectancies assume such values which make individuals indifferent between the two alternatives, because each provides the same consumption path over lifetime.

This is no longer true if asymmetric information, where prices are distorted by adverse selection, is introduced in our model. Then, under the assumption of price competition between annuity companies, the price for any contract is the same for both risk-groups, and in general only a situation where both groups buy the same type of second-period contract is feasible. Further, it turns out that the type of contract chosen to provide for the second period of retirement also affects the price of the first-period contract. In particular, we find that the two strategies have differing consequences for the welfare of the individuals, because they allow different consumption paths over the time of retirement: long-living individuals, who put more weight on consumption in the second period, prefer the regime when all individuals make sequential provision, while short-living individuals prefer the regime with simultaneous provision. Assuming that insurance companies can credibly commit in the working period to offer contracts at a pre-specified price, we find that only the former regime, favourable to the long-living individuals, represents a Bertrand-Nash equilibrium. This result is puzzling because empirical evidence shows that in fact term-limited contracts represent a small share of annuity contracts [see, e.g., Mitchell et al., 1999]. We discuss possible explanations for this puzzle in the concluding remarks.

\footnotetext{
3 Yagi and Nishigaki [1993] also employed a model with one working period and two periods of retirement in order to discuss optimal insurance demand of a representative individual. They showed that constant annuity payouts over time are inefficient, given that the individual rate of time preference differs from the interest rate. However, they did not consider the adverse-section problem and its impact on the existence of equilibria.

${ }^{4}$ With these life annuities, firms can separate individuals according to their life expectancy by a variation of the payouts over time. In fact, only a separating equilibrium (compare Rothschild and Stiglitz, 1976) can occur.

5 Townley and Boadway [1988] studied the functioning of the annuity market when individuals save out of their payouts from the limited-time pension contract. In contrast, we consider the case that they can buy a second annuity to provide for the remaining time.
} 
In the following Section 2 we introduce the basic model and show that either simultaneous or sequential annuity contracts are chosen. We characterise demand in both cases. In Section 3 we analyse the consequences of adverse selection for annuity prices and for the existence of an equilibrium. Moreover, we study welfare of the individuals. Section 4 provides a discussion of the results.

\section{Sequential and simultaneous demand for annuities}

Consider an economy with $\mathrm{K}$ individuals who live for a maximum of three periods $t=0,1,2$. In the working period $t=0$, each individual $i$ earns a fixed labour income $w_{0}$. At the end of period 0 she retires and lives for at most two further periods. Survival to the retirement period $t=1$ occurs with probability $\pi_{1}^{i}, 0<\pi_{1}^{i}<1$. In the same way, given that an individual is alive in period 1 , survival to period 2 occurs with probability $\pi_{2}^{i}, 0<\pi_{2}^{i}<1$.

Provision for old age can be made through three types of annuity contracts, which are offered by insurance companies:

- $A_{1}$ denotes the quantity of a contract, which is bought at a price $Q_{1}$ in working period 0 and offers an immediate payout $A_{1}$ in retirement period 1 .

- $A_{2}$ denotes the quantity of a contract, which is bought at a price $Q_{2}$ in retirement period 1 and offers an immediate payout $A_{2}$ in retirement period 2.

- $D_{2}$ denotes the quantity of a contract, which is bought at a price $R_{2}$ in working period 0 and offers a deferred payout $D_{2}$ in retirement period 2 .

That is, each type of contract offers payouts for one period of retirement, but they differ in the date of purchase and the waiting period for the payout to begin: provision for retirement period 1 is made through $A_{1}$, while provision for retirement period 2 can be made through $A_{2}$ (bought by those only, who survive to retirement period 1) and/or through $D_{2}$ (bought already in the working period). $Q_{1}, Q_{2}$ and $R_{2}$ are the corresponding prices (premiums, resp.) per unit of annuity payout; the reciprocal of each price represents the rate of return on each contract type.

We assume that the individuals have no bequest motive, which means that saving is not an attractive strategy for them to provide for old-age. This follows from the fact that the rate of return of annuities is higher than the interest rate, as annuities allow to avoid unintended bequests (see Yaari [1965]). Further, in order to concentrate on the design of the annuity contracts and to simplify the analysis, the assumption is made that no public pension system exists. The budget equation of an individual i for the working period 0 is

$$
c_{0}^{i}=w_{0}-Q_{1} A_{1}^{i}-R_{2} D_{2}^{i} .
$$

Moreover, given that individual $i$ is alive in the retirement period 1, she can spend an amount $Q_{2} A_{2}^{i}$ from her income $A_{1}^{i}$ in order to make additional provision for consumption in the retirement period 2, and consumes an amount $c_{1}^{i}$. This gives us the budget equations for the two retirement periods $t=1,2$ :

$$
\begin{aligned}
& c_{1}^{i}=A_{1}^{i}-Q_{2} A_{2}^{i}, \\
& c_{2}^{i}=A_{2}^{i}+D_{2}^{i} .
\end{aligned}
$$


Preferences over lifetime consumption of an individual $i$ are time-separable and are represented by expected utility with a per-period utility function $u$ depending on consumption. An individual $i$ is confronted with the following two-stage decision problem: in the working period 0 , she decides on the quantities $A_{1}^{i}$ and $D_{2}^{i}$ of annuities, thus on her consumption level in period 0 and on her income $w_{t}^{i}$ in each of the two retirement periods $t=1,2$. For this decision she takes into account her optimal annuity demand $A_{2}^{i}$ and her optimal consumption levels in periods 1 and 2, about which she will decide in period 1, given that then she is alive. Formally, this two-stage problem can be written as:

$$
t=0: \max u\left(c_{0}^{i}\right)+\pi_{1}^{i} \varphi^{i}\left(A_{1}^{i}, Q_{2}, D_{2}^{i}\right)
$$

s.t. $(2.1)$,

$$
t=1: \max u\left(c_{1}^{i}\right)+\pi_{2}^{i} u\left(c_{2}^{i}\right)
$$

s.t. (2.2) and (2.3),

where $\varphi^{i}\left(A_{1}^{i}, Q_{2}, D_{2}^{i}\right) \equiv \max _{c_{1}^{i}, c_{2}^{i}, A_{2}^{i}}\left\{u\left(c_{1}^{i}\right)+\pi_{2}^{i} u\left(c_{2}^{i}\right) \mid c_{1}^{i}=A_{1}^{i}-Q_{2} A_{2}^{i}, c_{2}^{i}=D_{2}^{i}+\right.$ $\left.A_{2}^{i}\right\}$.

Concerning the $A_{2}$-contract, we in fact assume that the individuals are informed about its price $Q_{2}$ already in the working period 0 , in other words, that the insurance companies can credibly commit to offer those contracts at a price $Q_{2}$ one period later. Otherwise $\varphi^{i}$ would not be well-defined. ${ }^{6}$ Further, we assume $u^{\prime}\left(c_{t}^{i}\right)>0, u^{\prime \prime}\left(c_{t}^{i}\right)<0$ and $\lim _{c \rightarrow 0} u^{\prime}(c)=\infty$. Notice that the specification of the decision problem means that the individuals do not discount future consumption for any reason other than risk aversion. $^{7}$

By inserting (2.1) into (2.4) and differentiating with respect to $A_{1}^{i}$ and $D_{2}^{i}$ as well as inserting (2.2) and (2.3) into (2.5) and differentiating with respect to $A_{2}^{i}$, we obtain the Kuhn-Tucker conditions of this maximization problem:

$$
\begin{gathered}
-Q_{1} u^{\prime}\left(w_{0}-Q_{1} A_{1}^{i}-R_{2} D_{2}^{i}\right)+\pi_{1}^{i} \frac{\partial \varphi^{i}\left(A_{1}^{i}, Q_{2}, D_{2}^{i}\right)}{\partial A_{1}^{i}}=0, \\
D_{2}^{i}>0 \text { and }-R_{2} u^{\prime}\left(w_{0}-Q_{1} A_{1}^{i}-R_{2} D_{2}^{i}\right)+\pi_{1}^{i} \frac{\partial \varphi^{i}\left(A_{1}^{i}, Q_{2}, D_{2}^{i}\right)}{\partial D_{2}^{i}}=0 \text { or } \\
D_{2}^{i}=0 \text { and }-R_{2} u^{\prime}\left(w_{0}-Q_{1} A_{1}^{i}-R_{2} D_{2}^{i}\right)+\pi_{1}^{i} \frac{\partial \varphi^{i}\left(A_{1}^{i}, Q_{2}, D_{2}^{i}\right)}{\partial D_{2}^{i}} \leqslant 0, \\
A_{2}^{i}>0 \text { and }-Q_{2} u^{\prime}\left(A_{1}^{i}-Q_{2} A_{2}^{i}\right)+\pi_{2}^{i} u^{\prime}\left(D_{2}^{i}+A_{2}^{i}\right)=0 \text { or } \\
A_{2}^{i}=0 \text { and }-Q_{2} u^{\prime}\left(A_{1}^{i}-Q_{2} A_{2}^{i}\right)+\pi_{2}^{i} u^{\prime}\left(D_{2}^{i}+A_{2}^{i}\right) \leqslant 0,
\end{gathered}
$$

\footnotetext{
${ }^{6}$ We leave it to the concluding section to discuss the consequences of relaxing this assumption, e.g. that the individuals are uncertain in the working period about the future price level $Q_{2}$, due to missing instruments of credible commitment by the firms.

7 To simplify notation, we do not include a time preference parameter explicitly in the utility function. To do so, would mean that a per-period discount factor enters (2.4) and (2.5) just in the same way as the survival probabilities. Nothing would change with the results.
} 
where by application of the Envelope Theorem

$$
\begin{aligned}
& \frac{\partial \varphi^{i}\left(A_{1}^{i}, Q_{2}, D_{2}^{i}\right)}{\partial A_{1}^{i}}=u^{\prime}\left(c_{1}^{i}\right), \\
& \frac{\partial \varphi^{i}\left(A_{1}^{i}, Q_{2}, D_{2}^{i}\right)}{\partial D_{2}^{i}}=\pi_{2}^{i} u^{\prime}\left(c_{2}^{i}\right) .
\end{aligned}
$$

Obviously, an individual $i$ always has a positive annuity demand $A_{1}^{i}$ for the first-period contract, since this is the only possibility to provide for first-period consumption. But she can decide either to buy the immediate annuity contract (i.e. $A_{2}^{i}>0, D_{2}^{i}=0$ ) or the deferred contract (i.e. $A_{2}^{i}=0, D_{2}^{i}>0$ ) or both kind of contracts (i.e. $A_{2}^{i}>0$, $D_{2}^{i}>0$ ) in order to make provision for consumption in the second retirement period. The following Lemma shows that the latter case is in general excluded.

Lemma 1. In general, it is not optimal for an individual $i$ to choose both $D_{2}^{i}>0$ and $A_{2}^{i}>0$. The inequality $Q_{1} Q_{2}>R_{2}\left(Q_{1} Q_{2}<R_{2}\right)$ implies $D_{2}^{i}>0$ and $A_{2}^{i}=0$ ( $A_{2}^{i}>0$ and $D_{2}^{i}=0$, resp.).

Proof: See the Appendix.

In order to receive one unit of payout in the second retirement period, the individual has to invest $Q_{2}$ units into the $A_{2}$-contract in the first retirement period and hence $Q_{1} Q_{2}$ units of income into the $A_{1}$-contract in the working period. On the other hand, $R_{2}$ units of income invested (in the working period) into the $D_{2}$-contract transforms into one unit of payout in the second retirement period. Therefore, the decisive relation is $Q_{1} Q_{2} \gtrless R_{2}$, whether provision for the second period of retirement is made through an $A_{2}$ - or a $D_{2}$-contract. Only in case of $Q_{1} Q_{2}=R_{2}$, the individuals would be indifferent between both types of contracts. For the remainder of this section we rule out this specific parameter constellation, but we distinguish between the two different situations whether an individual expresses annuity demand sequentially or simultaneously. In the first case of sequential annuity demand, the purchase of $A_{t}^{i}, t=1,2$, arises from a two-stage decision process, whose optimal solution is characterised by (2.6) and (2.8a). In the second case of simultaneous annuity demand, the purchased amounts $A_{1}^{i}, D_{2}^{i}$ are determined by the first-order conditions (2.6) and (2.7a).

The following two Lemmas characterise how prices and the survival probabilities influence annuity demand in both cases.

\section{Lemma 2.}

(i) In case of sequential annuity demand, i.e. $A_{1}^{i}>0, A_{2}^{i}>0, D_{2}^{i}=0$, we have

$$
\begin{aligned}
& \frac{\partial A_{1}^{i}\left(Q_{1}, Q_{2}\right)}{\partial Q_{1}}<0, \quad \frac{\partial A_{2}^{i}\left(Q_{1}, Q_{2}\right)}{\partial Q_{1}}<0, \\
& \frac{\partial A_{1}^{i}\left(Q_{1}, Q_{2}\right)}{\partial Q_{2}} \lesseqgtr 0, \quad \text { if } \varepsilon_{2}^{i} \lesseqgtr-1, \quad \frac{\partial A_{2}^{i}\left(Q_{1}, Q_{2}\right)}{\partial Q_{2}}<0,
\end{aligned}
$$

where $\varepsilon_{2}^{i}$ denotes the price elasticity of annuity demand $A_{2}^{i}$ for constant $A_{1}^{i}$. 
(ii) In case of simultaneous annuity demand, i.e. $A_{1}^{i}>0, A_{2}^{i}=0, D_{2}^{i}>0$, we have:

$$
\begin{aligned}
& \frac{\partial A_{1}^{i}\left(Q_{1}, R_{2}\right)}{\partial Q_{1}}<0, \quad \frac{\partial D_{2}^{i}\left(Q_{1}, R_{2}\right)}{\partial Q_{1}} \lesseqgtr 0 \quad \text { if } \eta_{1}^{i} \lesseqgtr-1, \\
& \frac{\partial A_{1}^{i}\left(Q_{1}, R_{2}\right)}{\partial R_{2}} \lesseqgtr 0 \quad \text { if } \eta_{2}^{i} \lesseqgtr-1, \quad \frac{\partial D_{2}^{i}\left(Q_{1}, R_{2}\right)}{\partial R_{2}}<0,
\end{aligned}
$$

where $\eta_{1}^{i}$ denotes the price elasticity of annuity demand $A_{1}^{i}$ for constant $D_{2}^{i}$ and $\eta_{2}^{i}$ the price elasticity of annuity demand $D_{2}^{i}$ for constant $A_{1}^{i}$.

\section{Proof: See Appendix.}

We find the usual result that demand for an annuity contract decreases, if its price rises. However, demand may but need not react in the same way, if the price of the other contract rises. The reasoning for the cross-price effects in case of sequential demand is the following: An increase in $Q_{1}$ reduces demand for the $A_{1}$-contract and hence income $w_{1}^{i}=A_{1}^{i}$ in retirement period 1 , out of which the $A_{2}$-contract has to be financed. The individual adapts to the decrease in $w_{1}^{i}$ (which would mean a reduction of $c_{1}^{i}$ in case of unchanged $A_{2}^{i}$ ) by decreasing demand for the $A_{2}$-contract (and thus consumption $c_{2}^{i}$ as well). The cross-price effect $\partial A_{1}^{i} / \partial Q_{2}$ follows from related arguments: In this case, it is essential how a change in $Q_{2}$ affects consumption $c_{1}^{i}$ holding $A_{1}^{i}$ constant for the moment. Note that, although $\partial A_{2}^{i} / \partial Q_{2}<0$, obviously, the expenditures $Q_{2} A_{2}^{i}$ for the $A_{2}$-contract and hence $c_{1}^{i}$ for fixed $A_{1}^{i}$ may de- or increase, depending on the price elasticity $\varepsilon_{2}^{i}$ of demand $A_{2}^{i}$ for fixed $w_{1}^{i}=A_{1}^{i}$. If $Q_{2}$ increases and $\varepsilon_{2}^{i}$ is larger than -1 , then $Q_{2} A_{2}^{i}$ increases, which means a reduction of $c_{1}^{i}$. Then it is optimal for the individual to shift part of the reduction to the working period 0 by decreasing $c_{0}^{i}$ and increasing demand for the $A_{1}$-contract. By the same argument, it is optimal for an individual to leave demand for the $A_{1}$-contract unchanged, if $\varepsilon_{2}^{i}=-1$, and to decrease demand $A_{1}^{i}$, if $\varepsilon_{2}^{i}<-1$.

Analogous considerations apply for the cross-price effects in the case of simultaneous demand: The relevant issue is how an increase of the prices $Q_{1}$ and $R_{2}$, resp., directly affects expenditures $Q_{1} A_{1}^{i}$ and $R_{2} D_{2}^{i}$, resp. (with demand for the other contract held constant). If expenditures increase $\left(\eta_{t}^{i}>-1\right)$, then part of the implied reduction of consumption $c_{0}^{i}$ in the working period is shifted to the respective other period through a reduction of the corresponding annuity demand; and analogous for elasticities $\eta_{t}^{i} \leqslant-1$.

\section{Lemma 3.}

(i) In case of sequential annuity demand, i.e. $A_{1}^{i}>0, A_{2}^{i}>0, D_{2}^{i}=0$, we have

$$
\begin{array}{ll}
\frac{\partial A_{1}^{i}\left(Q_{1}, Q_{2}\right)}{\partial \pi_{1}^{i}}>0, & \frac{\partial A_{2}^{i}\left(Q_{1}, Q_{2}\right)}{\partial \pi_{1}^{i}}>0, \\
\frac{\partial A_{1}^{i}\left(Q_{1}, Q_{2}\right)}{\partial \pi_{2}^{i}}>0, & \frac{\partial A_{2}^{i}\left(Q_{1}, Q_{2}\right)}{\partial \pi_{2}^{i}}>0 .
\end{array}
$$


(ii) In case of simultaneous annuity demand, i.e. $A_{1}^{i}>0, A_{2}^{i}=0, D_{2}^{i}>0$, we have

$$
\begin{array}{ll}
\frac{\partial A_{1}^{i}\left(Q_{1}, R_{2}\right)}{\partial \pi_{1}^{i}}>0, & \frac{\partial D_{2}^{i}\left(Q_{1}, R_{2}\right)}{\partial \pi_{1}^{i}}>0, \\
\frac{\partial A_{1}^{i}\left(Q_{1}, R_{2}\right)}{\partial \pi_{2}^{i}}<0, & \frac{\partial D_{2}^{i}\left(Q_{1}, R_{2}\right)}{\partial \pi_{2}^{i}}>0 .
\end{array}
$$

Proof: See Appendix.

We find that generally annuity demand reacts positively, if any probability of survival increases. However, there is an essential difference between the two cases, which concerns the cross effect of $\pi_{2}^{i}$ on the first-period contract. With sequential decisions, an increase of the probability of survival to the second period of retirement increases demand $A_{1}^{i}$, because this increase allows to buy more insurance for period 2 . On the other hand, with simultaneous decisions an increase of $\pi_{2}^{i}$ means that insurance for the first period of retirement is substituted by insurance for the second period of retirement. Note further that an increase in $\pi_{1}^{i}$ clearly increases the probability $\pi_{1}^{i} \pi_{2}^{i}$ of survival to the second period as well, hence demand for the second-period contracts rises in both cases.

\section{The market for time-limited annuities}

Having described the two possible strategies to provide for old age, namely through sequential and simultaneous annuity demand, we now study the implications of asymmetric information on the functioning of the annuity market. Let from now the otherwise identical individuals be divided into two groups $i=L, H$, characterised by different risks of a long life, i.e. by different probabilities of survival $\pi_{t}^{H}>\pi_{t}^{L}$ for $t=1,2$. Let $\gamma_{0}$ and $1-\gamma_{0}$, resp., denote the shares of the high-risk and low-risk individuals in period 0 , with $0<\gamma_{0}<1$.

\subsection{Perfect information}

First, as a point of reference we shortly consider the case that there is perfect information about the survival probabilities. Then, obviously, competition among the insurance firms ensures that each type of individuals receive their individually fair contracts, that is, expected payouts equal the price: $Q_{t}^{i}=\pi_{t}^{i}, t=1,2, R_{2}^{i}=\pi_{1}^{i} \pi_{2}^{i}, i=L, H$. Clearly, this implies that the annuity companies make zero expected profits.

Lemma 4. Given individually fair contracts, any individual is indifferent between choosing an $A_{2}$ - or $D_{2}$-contract for the second period of retirement. She chooses the same level of consumption in every period $t=0,1,2$.

Proof: The zero-profit conditions $Q_{t}^{i}=\pi_{t}^{i}, t=1,2$ and $R_{2}^{i}=\pi_{1}^{i} \pi_{2}^{i}$ imply $Q_{1}^{i} Q_{2}^{i}=$ $R_{2}^{i}, i=L, H$, which is the condition for indifference, as mentioned after Lemma 1. Springer 
Considering (2.6)-(2.10), one observes that the zero-profit conditions also imply $u^{\prime}\left(c_{0}^{i}\right)=u^{\prime}\left(c_{1}^{i}\right)=u^{\prime}\left(c_{2}^{i}\right)$, irrespective of the chosen contracts.

In a first-best world, where every individual can buy an annuity contract whose price is precisely adjusted to her life expectancy, it does not matter, which type of contract is chosen for provision for the second period of retirement.

\subsection{Asymmetric information and price competition}

However, in reality lack of information prevents the supply of first-best contracts. Therefore, we now turn to a more realistic setting by introducing asymmetric information into the model in the usual way: The probabilities $\pi_{t}^{i}$ and $\gamma_{0}$ are public information, known by the annuity companies. But it is the private information for each individual to know her type, i.e. her probability of survival. As a consequence, there is an adverse-selection problem in the annuity market.

Moreover, we assume that there is perfect competition among the annuity companies and that they cannot monitor whether consumers buy annuities from other insurance companies, which means that annuity contracts are nonexclusive. Thus, there is only competition about prices, which can be characterised as a third-best mechanism. ${ }^{8}$ This seems to be a reasonable assumption frequently made for the annuity market [see e.g. Pauly, 1974; Abel, 1986; Brugiavini, 1993; Walliser, 2000; Pech, 2004; Brunner and Pech, 2005]. It implies that firms fix the price of a contract and individuals can buy as many annuities of each contract as they want.

It follows that in equilibrium for each contract only one price, paid by both types of individuals, can exist. ${ }^{9}$ As a consequence, the first-best solution is unsustainable, because the individually fair prices of the $A_{1}-, A_{2}$ - and $D_{2}$-contract as defined above are lower for type- $L$ individuals than for type- $H$ individuals.

As both types of individuals buy the same contract, that is, pay the same price per unit of annuity payout, we can also conclude that only a situation, where both groups use the same type of contract in order to provide for the second period of retirement, can prevail, given that prices are such that $Q_{1} Q_{2} \gtreqless R_{2}$ :

Lemma 5. For any $Q_{1}, Q_{2}$ and $R_{2}$ : If $Q_{1} Q_{2}<(>) R_{2}$, both groups use the $A_{2}$ contract (the $D_{2}$-contract) to provide for the second period of retirement.

Proof: Price competition implies that only one price $Q_{1}$ for the first-period contract can exist. The criterion whether to choose the $A_{2}$-or the $D_{2}$-contract to provide for period 2 is $Q_{1} Q_{2} \gtrless R_{2}$ (see Lemma 1), which is the same for both groups.

\footnotetext{
${ }^{8}$ In this terminology, price and quantity competition, where firms offer a number of different contracts which specify both a price and a quantity, represents a second-best mechanism. As this generates the possibility of a separating equilibrium [see Rothschild and Stiglitz, 1976; Wilson, 1977], it allows a more efficient outcome in view of the information constraint. However, it requires that individuals can be restricted to buy at most one contract, which is regarded as being appropriate for some insurance markets, e.g. insurance against accidents, but not for the annuity market.

${ }^{9}$ That both types of individuals buy the same contract could be called a pooling situation. However, in a strict sense, this term refers to a framework where in principle the groups could be separated through appropriate instruments, e.g. through price and quantity competition.
} 
For this reason, we distinguish in the following between two different regimes, where all individuals demand either the sequential or the simultaneous second-period contract. (Obviously, a situation where both second-period contracts are traded, can occur only if $Q_{1} Q_{2}=R_{2}$. Then individuals are indifferent between the $A_{2}$-and the $D_{2}$-contract and they may choose one or the other contract. We analyse this case in Subsection 3.5.)

Sequential regime: $A_{1}^{i}>0, A_{2}^{i}>0, D_{2}^{i}=0 \quad$ for $i=L, H$,

Simultaneous regime: $A_{1}^{i}>0, A_{2}^{i}=0, D_{2}^{i}>0 \quad$ for $i=L, H$.

In the following a tilde refers to the sequential regime, while a bar refers to the simultaneous regime.

The extent of adverse selection in both regimes

As a next step we discuss, to which extent the adverse-selection problem matters in the two regimes, that is, whether individuals with a long life expectancy buy a larger amount of the different types of contracts.

\section{Lemma 6.}

(i) In the sequential regime, for any prices $Q_{1}, Q_{2}$, an individual with high survival probabilities demands larger quantities of both annuities than an individual with low survival probabilities, i.e. $\tilde{A}_{t}^{H}\left(Q_{1}, Q_{2}\right)>\tilde{A}_{t}^{L}\left(Q_{1}, Q_{2}\right), t=1,2$.

(ii) In the simultaneous regime, for any prices $Q_{1}, R_{2}$, an individual with high survival probabilities demands a larger quantity $D_{2}$ than an individual with low survival probabilities, i.e. $\bar{D}_{2}^{H}\left(Q_{1}, R_{2}\right)>\bar{D}_{2}^{L}\left(Q_{1}, R_{2}\right)$. The ratio of demand for the $A_{1}$ contract is undetermined.

Proof: Follows immediately from Lemma 3 and $\pi_{t}^{L}<\pi_{t}^{H}, t=1,2$.

If the problem of adverse selection is defined by the criterion that the ratio of aggregate group-H demand to aggregate group-L demand exceeds the ratio of group shares $\gamma_{0} /\left(1-\gamma_{0}\right)$, we find that this problem certainly occurs for both contracts in the sequential regime; in the simultaneous regime it occurs for the second-period contract, while for the first-period contract it is mitigated by the fact that an increase of $\pi_{2}^{i}$ decreases demand for the $A_{1}$-contract.

The above results concerning demand for the first-period contract in each regime indicate that the adverse-selection problem for the first-period contract is more severe in the sequential regime than in the simultaneous regime. We define the ratio of annuity demand of both risk types for the sequential first- and second-period contracts by $\tilde{\rho}_{t}\left(Q_{1}, Q_{2}\right) \equiv \tilde{A}_{t}^{H}\left(Q_{1}, Q_{2}\right) / \tilde{A}_{t}^{L}\left(Q_{1}, Q_{2}\right), t=1,2$, and the demandratio for the simultaneous first- and second-period contracts by $\bar{\rho}_{1}\left(Q_{1}, R_{2}\right) \equiv$ $\bar{A}_{1}^{H}\left(Q_{1}, R_{2}\right) / \bar{A}_{1}^{L}\left(Q_{1}, R_{2}\right)$ and $\bar{\rho}_{2}\left(Q_{1}, R_{2}\right) \equiv \bar{D}_{2}^{H}\left(Q_{1}, R_{2}\right) / \bar{D}_{2}^{H}\left(Q_{1}, R_{2}\right)$, resp. In the following, we restrict our analysis to the case that

$\tilde{\rho}_{1}\left(Q_{1}, Q_{2}\right)>\bar{\rho}_{1}\left(Q_{1}, R_{2}\right)$, for any $Q_{1}, Q_{2}$ and $R_{2}$ such that $Q_{1} Q_{2}=R_{2}$. 
That is, if prices are such that the individuals are indifferent between the sequential and the simultaneous regime, then the adverse-selection problem for the first-period contract is more severe in the simultaneous regime. Obviously, it depends on the individuals' preferences whether (3.1) holds. We can show that for expected utility, with per-period utility exhibiting a constant Arrow-Pratt coefficient of relative risk aversion $\sigma>0$ (abbreviated by CRRA utility in the following), i.e. with

$$
u\left(c_{t}^{i}\right)=\frac{\left(c_{t}^{i}\right)^{1-\sigma}-1}{1-\sigma}, \quad \text { for } t=0,1,2
$$

the Assumption (3.1) is indeed fulfilled.

Lemma 7. With CRRA utility, the relation $\tilde{\rho}_{1}\left(Q_{1}, Q_{2}\right)>\bar{\rho}_{1}\left(Q_{1}, R_{2}\right)$ holds, for any $Q_{1}, Q_{2}$ and $R_{2}$ such that $Q_{1} Q_{2}=R_{2}$.

Proof: See Appendix.

The intuitive reason for $\tilde{\rho}_{1}>\bar{\rho}_{1}$ is the following: In the simultaneous regime, annuity demand $\bar{A}_{1}^{i}$ for the first-period contract satisfies only the need for future consumption in period 1 . In contrast, in the sequential regime, annuity demand $\tilde{A}_{1}^{i}$ for the first-period contract has to satisfy the need for future consumption in both retirement periods 1 and 2 , since part of the payouts $\tilde{A}_{1}^{i}$ is used for the demand $\tilde{A}_{2}^{i}$. High-risk individuals choose a higher demand $\tilde{A}_{2}^{i}$ than low risk-individuals (see Lemma 5), which in turn intensifies adverse selection for the first-period contract.

Moreover, we can show that (3.1) is in fact equivalent to the opposite relation between the demand ratios of the first-period sequential contract and the second-period simultaneous contract.

Lemma 8. For any $Q_{1}, Q_{2}$ and $R_{2}$ such that $Q_{1} Q_{2}=R_{2}$ : If the ratio of the high- and low-risk types' annuity demand for the first-period sequential contract is higher than that for the first-period simultaneous contract, then the former ratio is lower than that for the second-period simultaneous contract, and vice versa. I.e., iff $\tilde{\rho}_{1}\left(Q_{1}, Q_{2}\right)>$ $\bar{\rho}_{1}\left(Q_{1}, R_{2}\right)$, then $\tilde{\rho}_{1}\left(Q_{1}, Q_{2}\right)<\bar{\rho}_{2}\left(Q_{1}, R_{2}\right)$.

Proof: See Appendix.

Intuitively, this equivalence follows from the fact that in the simultaneous regime there is a clear trade-off between demand for first-period and for second-period annuities. Moreover, with prices such that individuals are indifferent between the two regimes, the part of income $\mathrm{w}_{0}$ devoted to provision for both retirement periods is same in both regimes. This part is equal to $Q_{1} \tilde{A}_{1}$ in the sequential regime and equal to $Q_{1} \bar{A}_{1}+R_{2} \bar{D}_{2}$ in the simultaneous regime. Then, if the high-risk types buy relatively less of $\bar{A}_{1}$ than of $\tilde{A}_{1}$, they necessarily buy relatively more of $\bar{D}_{2}$ than of $\tilde{A}_{1}$. 


\section{Prices in both regimes}

The consequence of the over-representation of high-risk individuals among aggregate annuity demand is that in equilibrium insurance companies charge a price which is higher than the actuarially fair price corresponding to the average probability of survival of the population. In either regime, the respective prices are determined by the condition that, due to the assumption of perfect competition in the annuity market, the expected profits of each contract, bought by both groups $\mathrm{L}$ and $\mathrm{H}$, must be equal to zero.

Sequential regime. As $\pi_{1}^{i}$ is the expected payout rate of the $A_{1}$-contract for group $i$ and $\gamma_{0}$ is group H's share at the date of purchase, the zero-profit condition for the $A_{1}$-contract reads $\left(1-\gamma_{0}\right) \tilde{A}_{1}^{L}\left(Q_{1}, Q_{2}\right)\left(Q_{1}-\pi_{1}^{L}\right)+\gamma_{0} \tilde{A}_{1}^{H}\left(Q_{1}, Q_{2}\right)\left(Q_{1}-\pi_{1}^{H}\right)=0$, which can be rewritten by use of the definition of $\tilde{\rho}_{1}\left(Q_{1}, Q_{2}\right)$ and $\Gamma_{0} \equiv \gamma_{0} /\left(1-\gamma_{0}\right)$ as

$$
\left(Q_{1}-\pi_{1}^{L}\right)+\Gamma_{0} \tilde{\rho}_{1}\left(Q_{1}, Q_{2}\right)\left(Q_{1}-\pi_{1}^{H}\right)=0
$$

where $\Gamma_{0} \tilde{\rho}_{1}\left(Q_{1}, Q_{2}\right)$ is the ratio of aggregate demand of the high- and the low-risk groups. Since type- $H$ individuals have a higher probability to survive to retirement period 1, i.e. $\pi_{1}^{H}>\pi_{1}^{L}$, their share in period 1 will rise to

$$
\gamma_{1} \equiv \frac{\gamma_{0} \pi_{1}^{H}}{\gamma_{0} \pi_{1}^{H}+\left(1-\gamma_{0}\right) \pi_{1}^{L}}>\gamma_{0},
$$

while the share of type- $L$ individuals reduces to $\left(1-\gamma_{1}\right)$. Thus relatively more type- $H$ individuals exist to buy an $A_{2}$-contract. The expected payout rate for each group $i$ is $\pi_{2}^{i}$, thus by use of $\Gamma_{1} \equiv \gamma_{1} /\left(1-\gamma_{1}\right)$, the zero-profit condition for the $A_{2}$-contract can be written as

$$
\left(Q_{2}-\pi_{2}^{L}\right)+\Gamma_{1} \tilde{\rho}_{2}\left(Q_{1}, Q_{2}\right)\left(Q_{2}-\pi_{2}^{H}\right)=0
$$

Note that the zero-profit prices are simultaneously determined by the two equations (3.3) and (3.5), because in each equation the respective demand-ratio depends on both prices.

Simultaneous regime. In this regime, where the $A_{1}$-contract is supplemented by the $D_{2}$-contract, which is also bought in the working period 0 , the expected payout rate from the latter is $\pi_{1}^{i} \pi_{2}^{i}$ for each group $i$. Hence, the zero-profit prices are implicitly defined by the two zero-profit conditions:

$$
\begin{aligned}
\left(Q_{1}-\pi_{1}^{L}\right)+\Gamma_{0} \bar{\rho}_{1}\left(Q_{1}, R_{2}\right)\left(Q_{1}-\pi_{1}^{H}\right) & =0 \\
\left(R_{2}-\pi_{1}^{L} \pi_{2}^{L}\right)+\Gamma_{0} \bar{\rho}_{2}\left(Q_{1}, R_{2}\right)\left(R_{2}-\pi_{1}^{H} \pi_{2}^{H}\right) & =0 .
\end{aligned}
$$

One observes that a zero-profit situation in either regime is only sustainable, if profits are increasing in price. Otherwise the zero-profit contracts would be underbid Springer 
by lower-priced contracts creating positive profits. Thus, denoting the LHS's of (3.3), (3.5) by $\tilde{P}_{t}\left(Q_{1}, Q_{2}\right)$ and the LHS's of (3.6), (3.7) by $\bar{P}_{t}\left(Q_{1}, R_{2}\right), t=1,2$, we assume in the following, according to the usual assumption of production theory, that

$$
\begin{array}{ll}
\frac{\partial \tilde{P}_{1}\left(Q_{1}, Q_{2}\right)}{\partial Q_{1}}>0, & \frac{\partial \tilde{P}_{2}\left(Q_{1}, Q_{2}\right)}{\partial Q_{2}}>0, \\
& \text { for }\left(Q_{1}, Q_{2}\right) \in\left(\left(\pi_{1}^{L}, \pi_{2}^{L}\right),\left(\pi_{1}^{H}, \pi_{2}^{H}\right)\right), \\
\frac{\partial \bar{P}_{1}\left(Q_{1}, R_{2}\right)}{\partial Q_{1}}>0, & \frac{\partial \bar{P}_{2}\left(Q_{1}, R_{2}\right)}{\partial R_{2}}>0, \\
& \text { for }\left(Q_{1}, R_{2}\right) \in\left(\left(\pi_{1}^{L}, \pi_{1}^{L} \pi_{2}^{L}\right),\left(\pi_{1}^{H}, \pi_{1}^{H} \pi_{2}^{H}\right)\right) .
\end{array}
$$

Essentially, this means that with an increasing price of a contract the adverse-selection problem does not increase so much that an increase in expected payouts would outweigh increased revenues due to the higher price. Note that this assumption implies that there exists a unique pair of zero-profit prices in each regime.

Indeed, one finds that (3.8) and (3.9) are fulfilled for logarithmic utility (i.e. for CRRA utility with $\sigma=1$ ): This is immediate from the fact that for $\sigma=1$ all demand-ratios $\tilde{\rho}_{t}, \bar{\rho}_{t}, t=1,2$, are independent of prices (see (A51) and (A52) in the Appendix). For CRRA utility with $\sigma \neq 1$, a general proof that (3.8), (3.9) hold for all $\left(Q_{1}, Q_{2}\right),\left(Q_{1}, R_{2}\right)$, resp., cannot be given; however, numerical simulations for a wide range of parameter values have shown that (3.8), (3.9) hold.

The zero-profit prices, denoted by $\tilde{Q}_{1}, \tilde{Q}_{2}$ for the sequential regime and by $\bar{Q}_{1}, \bar{R}_{2}$ for the simultaneous regime, cannot be computed explicitly from the respective conditions. Nevertheless, if one takes the ratios $\Gamma_{t-1} \tilde{\rho}_{t}, \Gamma_{0} \bar{\rho}_{t}, t=1,2$ of aggregate demand of group $\mathrm{H}$ to that of group $\mathrm{L}$ as exogenous for the moment, one observes that the respective price is higher, the larger the respective demand-ratio. This gives us a first intuition for the results shown in the next Lemma.

Lemma 9. Suppose that in the simultaneous regime the demand-ratio of each contract is independent of the price of the other contract, i.e. $\partial \bar{\rho}_{1}\left(Q_{1}, R_{2}\right) / \partial R_{2}=0$ and $\partial \bar{\rho}_{2}\left(Q_{1}, R_{2}\right) / \partial Q_{1}=0$. Then

(i) the zero-profit price for the $A_{1}$-contract is lower in the simultaneous regime than in the sequential regime, i.e. $\bar{Q}_{1}<\tilde{Q}_{1}$,

(ii) the zero-profit price for the $D_{2}$-contract in the simultaneous regime is higher than the effective zero-profit price $\tilde{Q}_{1} \tilde{Q}_{2}$, which in the sequential regime has to be paid in the working period for the $A_{2}$-contract, i.e. $\bar{R}_{2}>\tilde{Q}_{1} \tilde{Q}_{2}$.

Proof: See Appendix.

The driving force behind the first result, $\bar{Q}_{1}<\tilde{Q}_{1}$, is the condition (3.1) which is fulfilled for standard preferences. It means that the overrepresentation of the highrisk types in demand for $A_{1}$-annuities is higher in the sequential regime than in the simultaneous regime $\left(\tilde{\rho}_{1}>\bar{\rho}_{1}\right.$, for any prices such that $\left.R_{2}=Q_{1} Q_{2}\right)$, because in the former regime, $A_{1}$-annuities also serve as an instrument for provision for retirement 
period 2. Therefore, if profits from the $A_{1}$-contract are zero with price $\tilde{Q}_{1}$ in the sequential regime, they will be positive in the simultaneous regime, if the price is the same (viz. $\tilde{Q}_{1}$ ). As a consequence, to have zero-profits in the simultaneous regime, the price must fall to $\bar{Q}_{1}<\tilde{Q}_{1}$.

To see why $\tilde{Q}_{1} \tilde{Q}_{2}<\bar{R}_{2}$, remember first that, in the sequential regime, $\tilde{Q}_{1} \tilde{Q}_{2}$ is the effective price which must be paid in the working period 0 for the $A_{2}$-contract. Intuitively, the reason why it is smaller than $\bar{R}_{2}$, the corresponding price in the simultaneous regime, again lies in the fact that in the former regime provision for period 2 is made via the first-period contract $A_{1}$, which is bought by the low-risk individuals to a larger extent than the $D_{2}$-contract (as $\tilde{\rho}_{1}<\bar{\rho}_{2}$ ). In other words, in the sequential regime the high-risk individuals, for which insurance for the second retirement period is especially important, benefit from being for the first period in a pool with the low-risk individuals, who put particular weight on insurance for this period, due to their short life expectancy. More precisely, the inequality $\tilde{Q}_{1} \tilde{Q}_{2}<\bar{R}_{2}$ is proved by showing that for $R_{2}=\tilde{Q}_{1} \tilde{Q}_{2}$ (which also means $\bar{\rho}_{2}=\tilde{\rho}_{2}$, see (A43) in the Appendix), the expected payoffs (in period 0) of both, the $D_{2}$ - and the $A_{2}$ - contract, are equal (viz. $\pi_{1}^{L} \pi_{2}^{L}+\pi_{1}^{H} \pi_{2}^{H} \Gamma_{0} \bar{\rho}_{2}$ ), while expected revenues are lower for the $D_{2}$ contract (viz. $\left.\tilde{Q}_{2}\left(\tilde{Q}_{1}+\tilde{Q}_{1} \Gamma_{0} \bar{\rho}_{2}\right)\right)$ than for the $A_{2}$-contract (viz. $\tilde{Q}_{2}\left(\pi_{1}^{L}+\pi_{1}^{H} \Gamma_{0} \tilde{\rho}_{2}\right)$, use $\Gamma_{1}=\Gamma_{0} \pi_{1}^{H} / \pi_{1}^{L}$ in (3.5) and multiply by $\left.\pi_{1}^{L}\right)$. Thus the former produces a loss with price $R_{2}=\tilde{Q}_{1} \tilde{Q}_{2}$, and it turns out that the low value of $\tilde{Q}_{1}$-being the zero-profit price for the sequential $A_{1}$-contract, determined by the "more profitable" demand-ratio $\tilde{\rho}_{1}<\bar{\rho}_{2}$-is responsible for this loss. As a consequence, the price $R_{2}$ must rise above $\tilde{Q}_{1} \tilde{Q}_{2}$ to guarantee zero-profits for the $D_{2}$-contract.

We obtain these two results unambiguously under the assumption that the demandratios in the simultaneous regime are independent of the price of the other contract, because this implies that expected profits for each contract do not depend on the price of the other contract. Obviously, this is fulfilled under logarithmic utility $(\sigma=1)$, because the cross-price effect of demand for the simultaneous contracts are zero (as $\eta_{t}^{i}=-1, t=1,2$, see Lemma 2).

However, when allowing for cross-price effects on the demand-shares, profits of each simultaneous contract do depend on both prices $Q_{1}, R_{2}$. Obviously, if the crossprice effects are sufficiently small, the own-price effects dominate and the results of Lemma 9 maintain. With CRRA utility and $\sigma \neq 1$, non-zero cross-price effects occur, but when running simulations for CRRA utility for a wide range of parameter values we found that indeed the cross-price effects are sufficiently small and $\tilde{Q}_{1}>\bar{Q}_{1}$ and $\tilde{Q}_{1} \tilde{Q}_{2}<\bar{R}_{2}$ still hold.

Note that the assumption that different risk types are defined by different probabilities of survival to both periods, $\pi_{t}^{H}>\pi_{t}^{L}, t=1,2$, is crucial for the results obtained in Lemma 9. To illustrate this, we consider the case (i) with $\pi_{1} \equiv \pi_{1}^{H}=\pi_{1}^{L}$ and $\pi_{2}^{H}>\pi_{2}^{L}$ and the case (ii) with $\pi_{1}^{H}>\pi_{1}^{L}$ and $\pi_{2} \equiv \pi_{2}^{H}=\pi_{2}^{L}$. In case (i), when risks differ only in the second retirement period, the relation (3.1), i.e. $\tilde{\rho}_{1}\left(Q_{1}, Q_{2}\right)>$ $\bar{\rho}_{1}\left(Q_{1}, R_{2}\right)$, if $R_{2}=Q_{1} Q_{2}$, follows immediately for any arbitrary utility function (as $\partial \tilde{A}_{1}^{i}\left(Q_{1}, Q_{2}\right) / \partial \pi_{2}^{i}>0$ and $\partial \bar{A}_{1}^{i}\left(Q_{1}, R_{2}\right) / \partial \pi_{2}^{i}<0$, see Lemma 3$)$. However, although each group buys a different amount of first-period annuities in either regime, they are offered at the same zero-profit price $\bar{Q}_{1}=\tilde{Q}_{1}=\pi_{1}$, as $\pi_{1}$ is the expected payout for both risk-types. The second important implication of $\pi_{1}^{H}=\pi_{1}^{L}$ is that the share of 
group $\mathrm{H}$ does not rise from period 0 to period 1, i.e. $\gamma_{1}=\gamma_{0}$ and $\Gamma_{1}=\Gamma_{0}$. From this, together with $\bar{Q}_{1}=\tilde{Q}_{1}=\pi_{1}$, it follows that in this case a price $\bar{R}_{2}=\tilde{Q}_{1} \tilde{Q}_{2}$ produces zero-profits for the $D_{2}$-contract. ${ }^{10}$

Next consider case (ii), when individuals only differ in their survival probability to the first period of retirement. As $\partial \tilde{A}_{1}^{i}\left(Q_{1}, Q_{2}\right) / \partial \pi_{1}^{i}>0$ and $\partial \bar{A}_{1}^{i}\left(Q_{1}, R_{2}\right) / \partial \pi_{1}^{i}>0$, while $\pi_{2} \equiv \pi_{2}^{H}=\pi_{2}^{L}$, the Assumption (3.1) of a higher demand-ratio for the $A_{1}$ contract in the sequential regime may, but need not hold. Indeed, under CRRA-utility (3.2) we have $\tilde{\rho}_{1}\left(Q_{1}, Q_{2}\right)=\bar{\rho}_{1}\left(Q_{1}, R_{2}\right)$, if $R_{2}=Q_{1} Q_{2}$ (which is immediate from (A38) in the Appendix) and consequently also $\tilde{\rho}_{1}\left(Q_{1}, Q_{2}\right)=\bar{\rho}_{2}\left(Q_{1}, R_{2}\right)$ (see (A41) in the Appendix). This equality of the demand-ratios in case of CRRA-utility is responsible for the result that also in case (ii) the zero-profit prices are the same in either regime, specifically we have: $\bar{Q}_{1}=\tilde{Q}_{1}, \bar{R}_{2}=\tilde{Q}_{1} \tilde{Q}_{2}$ and $\tilde{Q}_{2}=\pi_{2} \cdot{ }^{11}$ However, note that in this case (ii) it ultimately depends on the specifics of the per-period utility function whether $\tilde{\rho}_{1}\left(Q_{1}, Q_{2}\right) \gtreqless \bar{\rho}_{1}\left(Q_{1}, R_{2}\right)$, given $R_{2}=Q_{1} Q_{2}$, which is decisive for $\bar{Q}_{1} \lesseqgtr \tilde{Q}_{1}$ and $\bar{R}_{2} \lesseqgtr \tilde{Q}_{1} \tilde{Q}_{2}$. Yet in the former case (i), where individuals differ only in their survival probability to the second period, the result of equal prices in both regimes holds generally for any per-period utility function.

\subsection{Equilibrium}

Now we turn to an analysis of whether one or both of the two regimes constitute an equilibrium. To derive definite results, we consider the standard case that the inequalities $\tilde{Q}_{1}>\bar{Q}_{1}$ and $\tilde{Q}_{1} \tilde{Q}_{2}<\bar{R}_{2}$ are fulfilled. We call a set of contracts an equilibrium in the sense of Bertrand-Nash, if together with annuity demand of both groups $i=L, H$ the respective zero-profit condition for each contract is fulfilled and if no other contract exists, which is preferred by at least one group $i \in L, H$ and which allows a nonnegative profit.

Proposition 1. The sequential contracts with prices $\tilde{Q}_{1}, \tilde{Q}_{2}$ represent an equilibrium.

Proof: If the $A_{1}$-contract were offered at a price $Q_{1}<\tilde{Q}_{1}$, both groups would buy that and the insurance company would make a loss, as $\tilde{P}_{1}\left(\tilde{Q}_{1}, \tilde{Q}_{2}\right)=0$ and $\partial \tilde{P}_{1}\left(\tilde{Q}_{1}, \tilde{Q}_{2}\right) / \partial Q_{1}>0$. By the same argument (i.e. $\tilde{P}_{2}\left(\tilde{Q}_{1}, \tilde{Q}_{2}\right)=0$ and $\partial \tilde{P}_{2}\left(\tilde{Q}_{1}\right.$, $\left.\left.\tilde{Q}_{2}\right) / \partial Q_{2}>0\right)$, an insurance company offering an $A_{2}$-contract with price $Q_{2}<\tilde{Q}_{2}$ would make a loss. Finally, if an alternative $D_{2}$-contract with a price $R_{2}<\tilde{Q}_{1} \tilde{Q}_{2}$ was offered, again both groups would buy that contract (see Lemma 1) and the insurance company would make a loss. This follows from the fact that $\bar{P}_{2}\left(\tilde{Q}_{1}, \tilde{R}_{2}\right)<0$, where $\tilde{R}_{2} \equiv \tilde{Q}_{1} \tilde{Q}_{2}$, and $\partial \bar{P}_{2}\left(Q_{1}, R_{2}\right) / \partial R_{2}>0$ (see Proof of Lemma 9 ).

Proposition 2. The simultaneous contracts with prices $\bar{Q}_{1}, \bar{R}_{2}$ do not constitute an equilibrium.

\footnotetext{
${ }^{10}$ This is immediate from determining $\bar{P}_{2}\left(Q_{1}, R_{2}\right)$ for $Q_{1}=\pi_{1}$ and $R_{2}=\pi_{1} \tilde{Q}_{2}$ and by use of (3.5), remembering that $\bar{\rho}_{2}=\tilde{\rho}_{2}$ for $R_{2}=\tilde{Q}_{1} \tilde{Q}_{2}$.

${ }^{11}$ With these prices, the profit formulas (A42) and (A46) (in the Appendix) of the simultaneous-regime contracts can immediately be seen to yield zero.
} 
Proof: Given the simultaneous contracts with prices $\bar{Q}_{1}, \bar{R}_{2}$, an insurance company can additionally offer a sequential $A_{2}$-contract at some price $Q_{2}<\bar{R}_{2} / \bar{Q}_{1}$. At this price, both risk types would buy the $A_{2}$-contract (see Lemma 1 ) and firms would make a nonnegative profit. To show the latter, we proceed analogously as in the Proof of Lemma 9 and evaluate profits $\tilde{P}_{2}\left(Q_{1}, Q_{2}\right)$, the LHS of (3.5), at $\bar{Q}_{1}, \bar{Q}_{2} \equiv \bar{R}_{2} / \bar{Q}_{1}$ : We write (use $\Gamma_{1}=\Gamma_{0} \pi_{1}^{H} / \pi_{1}^{L}$ and $\bar{\rho}_{2}\left(\bar{Q}_{1}, \bar{R}_{2}\right)=\tilde{\rho}_{2}\left(\bar{Q}_{1}, \bar{Q}_{2}\right)$ )

$\tilde{P}_{2}\left(\bar{Q}_{1}, \bar{Q}_{2}\right)=\frac{1}{\pi_{1}^{L}}\left[\bar{Q}_{2}\left(\pi_{1}^{L}+\pi_{1}^{H} \Gamma_{0} \bar{\rho}_{2}\left(\bar{Q}_{1}, \bar{R}_{2}\right)\right)-\left(\pi_{1}^{L} \pi_{2}^{L}+\pi_{1}^{H} \pi_{2}^{H} \Gamma_{0} \bar{\rho}_{2}\left(\bar{Q}_{1}, \bar{R}_{2}\right)\right)\right.$,

which by use of (3.7) with $R_{2}=\bar{Q}_{1} \bar{Q}_{2}$ can be transformed to

$$
\tilde{P}_{2}\left(\bar{Q}_{1}, \bar{Q}_{2}\right)=\left(\bar{Q}_{2} / \pi_{1}^{L}\right)\left[-\left(\bar{Q}_{1}-\pi_{1}^{L}\right)-\left(\bar{Q}_{1}-\pi_{1}^{H}\right) \Gamma_{0} \bar{\rho}_{2}\left(\bar{Q}_{1}, \bar{R}_{2}\right)\right]
$$

and further, by use of (3.3), to

$$
\tilde{P}_{2}\left(\bar{Q}_{1}, \bar{Q}_{2}\right)=\left(\bar{Q}_{2} / \pi_{1}^{L}\right)\left(\bar{Q}_{1}-\pi_{1}^{H}\right) \Gamma_{0}\left[\tilde{\rho}_{1}\left(\bar{Q}_{1}, \bar{Q}_{2}\right)-\bar{\rho}_{2}\left(\bar{Q}_{1}, \bar{R}_{2}\right)\right]
$$

One observes from the last equation that $\tilde{P}_{2}\left(\bar{Q}_{1}, \bar{Q}_{2}\right)>0$, as $\bar{Q}_{1}<\pi_{1}^{H}$ and $\tilde{\rho}_{1}\left(\bar{Q}_{1}, \bar{Q}_{2}\right)<\bar{\rho}_{2}\left(\bar{Q}_{1}, \bar{R}_{2}\right)$. By continuity, we still have $\tilde{P}_{2}\left(\bar{Q}_{1}, Q_{2}\right) \geq 0$, i.e. nonnegative profits, at some price $Q_{2}<\bar{R}_{2} / \bar{Q}_{1}$, sufficiently close to $\bar{Q}_{2}$.

Intuitively there are two reasons why the sequential regime with prices $\tilde{Q}_{1}, \tilde{Q}_{2}$ constitutes an equilibrium: (i) From the above results we know that the sequential regime allows provision for the second retirement period at a lower price. Thus, it is plausible that no better $D_{2}$-contract can be offered without making a loss. (ii) No lower price than $\tilde{Q}_{1}$ can be granted for the $A_{1}$-contract, in view of the fact that individuals use part of the returns from this $A_{1}$-contract to provide for the second period via the $A_{2}$-contract.

Conversely, the simultaneous regime with prices $\bar{Q}_{1}, \bar{R}_{2}$ is not an equilibrium, because firms can additionally offer an $A_{2}$-contract at a price $Q_{2}<\bar{R}_{2} / \bar{Q}_{1}$, which combined with the existing $A_{1}$-contract with price $\bar{Q}_{1}$ allows provision for both retirement periods at a lower price. (Obviously however, the existing $A_{1}$-contract with return $\bar{Q}_{1}$ would make a loss in this case, because $\tilde{Q}_{1}$ is the lowest price compatible with sequential contracts.)

\subsection{Welfare analysis}

In a further step of our analysis, we study welfare of both types of individuals $i=$ $L, H$ in the two regimes, in order to find out whether the equilibrium outcomethe sequential regime with prices $\tilde{Q}_{1}, \tilde{Q}_{2}$-is a favourable solution for one or both risk groups, compared to the simultaneous regime with prices $\bar{Q}_{1}, \bar{R}_{2}$. Again, for the upcoming analysis we concentrate on the standard case that the inequalities $\tilde{Q}_{1}>\bar{Q}_{1}$ and $\tilde{Q}_{1} \tilde{Q}_{2}<\bar{R}_{2}$ hold. Moreover, we assume that the expected utility function for lifetime consumption, $u\left(c_{0}^{i}\right)+\pi_{1}^{i} u\left(c_{1}^{i}\right)+\pi_{1}^{i} \pi_{2}^{i} u\left(c_{2}^{i}\right)$, is homothetic, as it is indeed the case with per-period CRRA utility (3.2). 
Lemma 10. With CRRA utility, we have:

(i) In either regime, the ratio of second-period to first-period consumption of risk type $H$ is higher than that of risk type $L$, i.e. $\tilde{c}_{2}^{H} / \tilde{c}_{1}^{H}>\tilde{c}_{2}^{L} / \tilde{c}_{1}^{L}$ and $\bar{c}_{2}^{H} / \bar{c}_{1}^{H}>\bar{c}_{2}^{L} / \bar{c}_{1}^{L}$ for any prices.

(ii) For the zero-profit prices the ratio of second-period to first-period consumption of any risk-type $i=L, H$ is higher in the sequential regime than in the simultaneous regime, i.e. $\tilde{c}_{2}^{i} / \tilde{c}_{1}^{i}>\bar{c}_{2}^{i} / \bar{c}_{1}^{i}$, for $i=L, H$.

Proof: See Appendix.

These results are illustrated graphically (see Figs. 1 and 2 ) by drawing the indifference curves and budget lines in the $\left(c_{1}^{i}, c_{2}^{i}\right)$-space, where we assume $c_{0}^{i}$ to be fixed at the optimal value, indicated by a tilde for the sequential regime and a bar for the simultaneous regime. The budget lines, abbreviated by $\widetilde{B L}^{i}$ for the sequential regime and by $\overline{B L}^{i}$ for the simultaneous regime, are obtained by eliminating $A_{1}^{i}, A_{2}^{i}$ and $A_{1}^{i}, D_{2}^{i}$, resp., and combining (2.1)-(2.3), where $D_{2}^{i}=0$ and $A_{2}^{i}=0$, resp.:

$$
\begin{aligned}
& \widetilde{B L}^{i}: w_{0}-\tilde{c}_{0}^{i}=\tilde{Q}_{1} c_{1}^{i}+\tilde{Q}_{1} \tilde{Q}_{2} c_{2}^{i}, \\
& \overline{B L}^{i}: w_{0}-\bar{c}_{0}^{i}=\bar{Q}_{1} c_{1}^{i}+\bar{R}_{2} c_{2}^{i},
\end{aligned}
$$

$\widetilde{B L}^{i}$ describes the feasible consumption bundles $\left(c_{1}^{i}, c_{2}^{i}\right)$ for an individual $i$ who invests the optimal amount $w_{0}-\tilde{c}_{0}^{i}=\tilde{Q}_{1} \tilde{A}_{1}^{i}$ into the first-period contract. She can consume

Fig. 1 Retirement consumption in the sequential regime

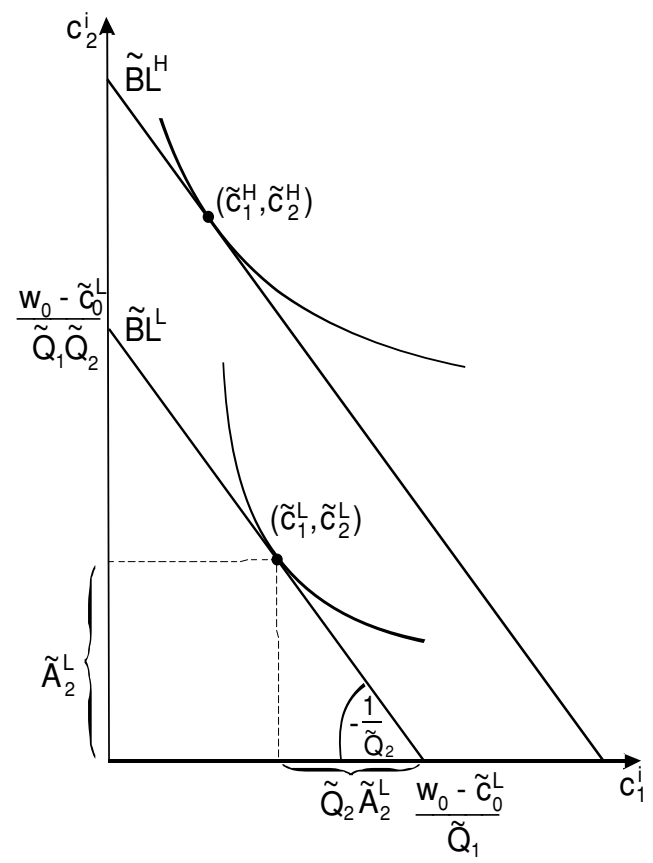




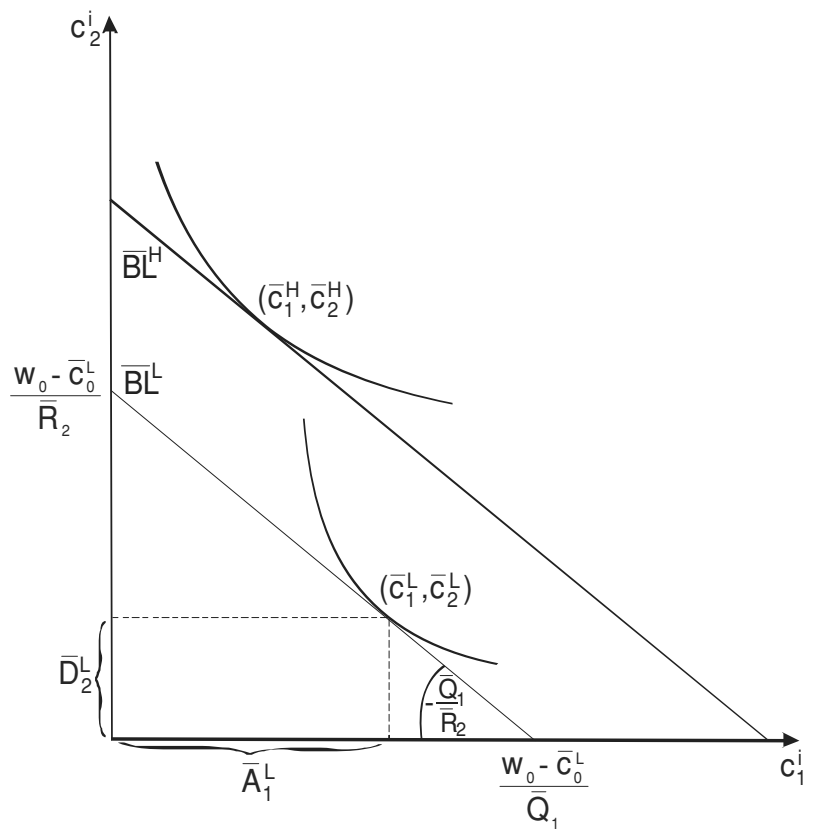

Fig. 2 Retirement consumption in the simultaneous regime

all payouts $c_{1}^{i}=\left(w_{0}-\bar{c}_{0}^{i}\right) / \tilde{Q}_{1}$ in period 1 or transform part of it into second-period consumption, by buying the sequential second-period contract at price $\tilde{Q}_{2}$. If she transforms everything, then $c_{2}^{i}=\left(w_{0}-\tilde{c}_{0}^{i}\right) /\left(\tilde{Q}_{1} \tilde{Q}_{2}\right)$ results. Thus, the slope of $\widetilde{B L}^{i}$ is $-1 / \tilde{Q}_{2}$ and it is the same for each risk type $L$ and $H$. On the other hand, $\overline{B L}^{i}$ represents all feasible consumption bundles for an individual $i$ who invests the optimal amount $w_{0}-\bar{c}_{0}^{i}$ into the $A_{1}$ - and $D_{2}$-contracts. Hence, in this regime, the trade-off (the slope of $\overline{B L}^{i}$ ) between consumption in period 1 and in period 2 is $-\bar{Q}_{1} / \bar{R}_{2}$ for both risk types $i=L, H$, which is the price ratio for one unit of payout in the second and in the first retirement period. In either regime, an individual $i$ chooses $\left(c_{1}^{i}, c_{2}^{i}\right)$ by maximizing $u\left(c_{1}^{i}\right)+\pi_{2}^{i} u\left(c_{2}^{i}\right)$ subject to respective budget line.

A comparison of the budget lines in both regimes demonstrates that for any type $i=L, H, \overline{B L}^{i}$ is flatter than $\widetilde{B L}^{i}$, because $\bar{Q}_{1} \tilde{Q}_{2}<\bar{R}_{2}$ due to Lemma 9 . This inequality is decisive for the distribution of consumption over the retirement periods, which under homothetic preferences, is independent of the optimal consumption in the working period 0: As the relative price for second-period consumption is lower in the sequential regime, relatively more consumption is postponed to the second period than in the simultaneous regime.

On the other hand, a comparison of the indifference curves in each regime shows that at any combination $\left(c_{1}^{i}, c_{2}^{i}\right)$ the slope $u^{\prime}\left(c_{1}^{i}\right) /\left(\pi_{2}^{i} u^{\prime}\left(c_{2}^{i}\right)\right)$ of the indifference curve is steeper for a type- $L$ individual than for a type- $H$ individual, as $\pi_{2}^{L}<\pi_{2}^{H}$. This property is responsible for the result that in each regime relative consumption $c_{2}^{i} / c_{1}^{i}$ of type $H$ is larger than that of type $L$. With homothetic preferences, this again holds irrespectively of what amount $w_{0}-c_{0}^{i}, i=L, H$ is invested into old-age provision. 悬 Springer 
(Obviously, in general optimal consumption in period 0 will be larger for the shortliving individuals than for the long-living ones in each regime, hence their budget lines are below those of the long-living, as drawn in Figs. 1 and 2.) The result conforms with intuition: Due to their higher survival probability to period 2, high-risk individuals value second-period consumption more than low-risk individuals; thus in either regime they postpone relatively more consumption to period 2 than low-risk individuals.

With these preparations, we now analyse welfare in the two regimes. There are the following possibilities: either both types are better off with one of the two regimes or each regime benefits a different type. With the properties established in Lemma 10, we can show that in the latter case there is a unique ranking:

Proposition 3. Let the ratio of second-to first-period consumption be larger for an individual of type $H$ than for an individual of type $L$. Then, if both risk types are differently affected by the two regimes, an individual of type $L$ is better off in the simultaneous regime with prices $\bar{Q}_{1}, \bar{R}_{2}$, while an individual of type $H$ is better off in the sequential regime with prices $\tilde{Q}_{1}, \tilde{Q}_{2}$.

Proof: It suffices to show that if type $H$ is better off in the simultaneous regime than in the sequential regime, this implies the same ordering for type $L$ as well. To see this, note that a change from the sequential to the simultaneous regime means that in the general budget equation,

$$
c_{0}^{i}+\beta_{1} c_{1}^{i}+\beta_{2} c_{2}^{i}=w_{0}
$$

the price $\beta_{1}$ of the first-period consumption decreases from $\beta_{1}=\tilde{Q}_{1}$ to $\beta_{1}=\bar{Q}_{1}$, while the price $\beta_{2}$ of the second-period consumption increases from $\beta_{2}=\tilde{Q}_{1} \tilde{Q}_{2}$ to $\beta_{2}=\bar{R}_{2}$.

We introduce the indirect utility function $v^{i}\left(\beta_{1}, \beta_{2}\right), i=L, H$, and consider the welfare effect of a marginal decrease in $\beta_{1}$ and an increase in $\beta_{2}$, (i.e., $d \beta_{1}<0$, $\left.d \beta_{2}>0\right)$. We have

$$
\begin{aligned}
d v^{i} & =\partial v^{i} / \partial \beta_{1} \cdot d \beta_{1}+\partial v^{i} / \partial \beta_{2} \cdot d \beta_{2} \\
& =\left(-c_{1}^{i} d \beta_{1}-c_{2}^{i} d \beta_{2}\right) \lambda,
\end{aligned}
$$

by Roy's Lemma, with $\lambda>0$ denoting the marginal utility of income. Obviously, in case that $d v^{H} \geq 0$, thus $-c_{1}^{H} d \beta_{1}-c_{2}^{H} d \beta_{2} \geq 0$, we also have $-c_{1}^{L} d \beta_{1}-c_{2}^{L} d \beta_{2}>0$ and $d v^{L}>0$, because $c_{2}^{H} / c_{1}^{H}>c_{2}^{L} / c_{1}^{L}$ and $\mathrm{d} \beta_{1}<0, d \beta_{2}>0$. As a result, a marginal change of the prices in favour of type $\mathrm{H}$ is also in favour of type L. Obviously, this reasoning holds also along the whole path from $\left(\tilde{Q}_{1}, \tilde{Q}_{1} \tilde{Q}_{2}\right)$ to $\left(\bar{Q}_{1}, \bar{R}_{2}\right)$.

This result conforms with what one expects in view of the relation between the prices in the two regimes, found in Lemma 9: If neither of the regimes is Pareto superior to the other, the simultaneous regime is favourable to the short-living individuals because it allows a cheaper provision for the first period of retirement, which is particularly important for them. In contrast, the relatively lower price for second-period provision in the sequential regime is favourable for the long-living individuals. 


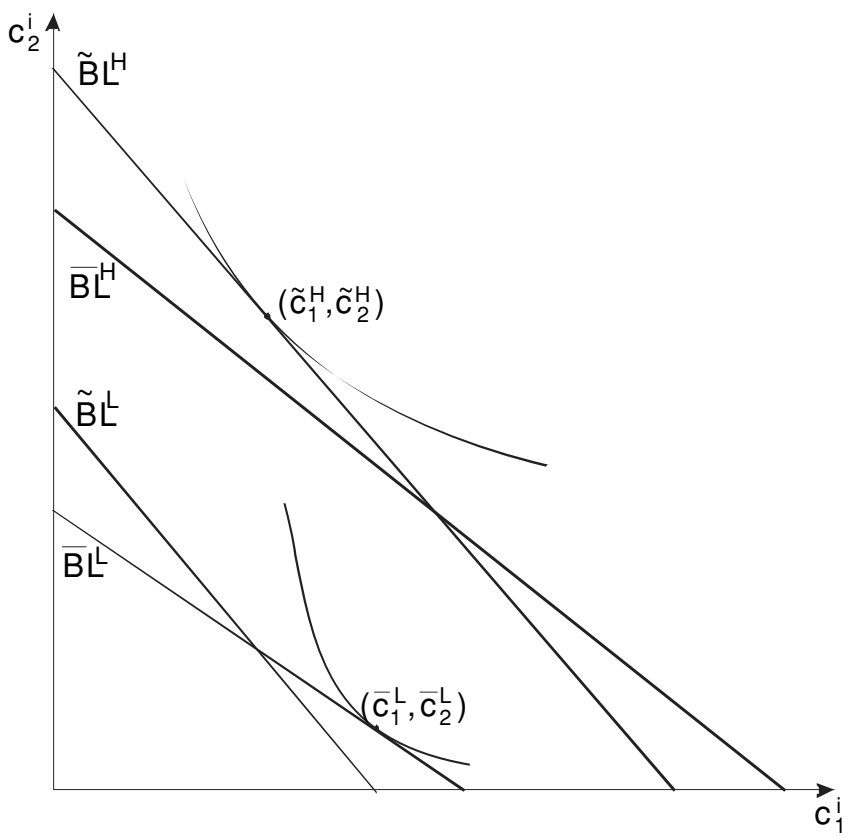

Fig. 3 Retirement consumption when the types prefer different regimes

Such a situation is illustrated in Fig. 3, where the budget lines (3.10) and (3.11) of both types of individuals $i=L, H$, restricting consumption in retirement, are drawn. Essential for the result of Proposition 3 is that at any combination $\left(c_{1}^{i}, c_{2}^{i}\right)$ the slope $-u^{\prime}\left(c_{1}^{i}\right) /\left(\pi_{2}^{i} u^{\prime}\left(c_{2}^{i}\right)\right)$ of the indifference curve is steeper for a type- $L$ individual than for a type- $H$ individual, as $\pi_{2}^{L}<\pi_{2}^{H}$. Then a situation as depicted in Fig. 3 may arise, where the optimum consumption point of type- $L$ individuals, given the simultaneous regime, provides higher welfare than the sequential regime, and vice versa for the type- $H$ individuals. ${ }^{12}$

One can indeed show that with logarithmic utility the two types of individuals are differently affected by the two regimes. The important property, which facilitates the analysis, is that for logarithmic utility, the expenditures for annuities in the working period do not depend on prices, and it is optimal for an individual to invest the same amount $\tilde{Q}_{1} \tilde{A}_{1}^{i}=\bar{Q}_{1} \bar{A}_{1}^{i}+\bar{R}_{2} \bar{D}_{2}^{i}, i=L, H$, into old-age provision in each regime. ${ }^{13}$

Proposition 4. Under logarithmic utility, an individual of type $L$ is better off in the simultaneous regime with prices $\bar{Q}_{1}, \bar{R}_{2}$, while an individual of type $H$ is better off in the sequential regime with prices $\tilde{Q}_{1}, \tilde{Q}_{2}$.

Proof: See Appendix.

\footnotetext{
${ }^{12}$ To be precise, it should be noted that the indifference curves through $\left(\tilde{c}_{1}^{H}, \tilde{c}_{2}^{H}\right)$ and $\left(\bar{c}_{1}^{L}, \bar{c}_{2}^{L}\right)$, resp., will move with a regime change, because in general $c_{0}^{i}$ will not be the same in the two regimes.

${ }^{13}$ Thus, also $\tilde{c}_{0}^{i}=\bar{c}_{0}^{i}, i=L, H$ in this case. 
As one can show, logarithmic utility implies that, irrespective of the regime, the optimal combination for a type- $L$ individual is to the right of the point of intersection of her budget lines $\widetilde{B L}^{L}$ and $\overline{B L}^{L}$, while the optimal consumption bundle for a type- $H$ individual lies to the left of the intersection of $\widetilde{B L}^{H}$ and $\overline{B L}^{H}$. Consequently, since the simultaneous regime allows higher consumption possibilities to the right of the point of intersection, it is preferred by a type- $L$ individual. The opposite holds for a type- $H$ individual.

For CRRA utility with $\sigma \neq 1$, a general proof cannot be given, because the zeroprofit prices cannot be computed explicitly (as it can be done for logarithmic utility). However, we ran numerical simulations using CRRA utility and $\sigma \neq 1$ for a wide range of parameter values and found the same result as proved for logarithmic utility: No regime is Pareto dominant, instead the sequential regime favours type- $H$ individuals, while it disadvantages type- $L$ individuals.

\subsection{The "all-markets-open" regime ${ }^{14}$}

In this final Subsection we want to investigate the possible existence of an equilibrium in which all markets are open, i.e. where both types of second-period contracts are traded in addition to the first-period contract (for which - due to price competitiononly a single price can exist). Obviously, such an equilibrium can only prevail, if prices are such that $Q_{1} Q_{2}=R_{2}$, and the individuals, being indifferent between sequential and simultaneous annuity demand, choose different strategies to provide for the second period of retirement.

We assume in the following that in case of indifference between the two secondperiod contracts, some share $\tilde{\beta}_{i}$ of each group $i=L, H$ decides (in the working period 0 !) to postpone their second-period provision to period 1 through the purchase of the sequential second-period contract, while a share $\left(1-\tilde{\beta}_{i}\right)$ of each group provides for the second period through the immediate purchase of the simultaneous second-period contract. We keep the assumption of price competition among the annuity companies, hence expected profits of all contracts have to be zero. Then a possible candidate for an equilibrium, in which all markets are open, is characterised by the zero-profit conditions for the $A_{1^{-}}, A_{2}$ - and $D_{2}$-contracts, resp., i.e. by

$$
\begin{aligned}
& \left(Q_{1}-\pi_{1}^{L}\right)\left[\tilde{\beta}_{L} \tilde{A}_{1}^{L}\left(Q_{1}, Q_{2}\right)+\left(1-\tilde{\beta}_{L}\right) \bar{A}_{1}^{L}\left(Q_{1}, R_{2}\right)\right]+\left(Q_{1}-\pi_{1}^{H}\right) \Gamma_{0}\left[\tilde{\beta}_{H} \tilde{A}_{1}^{H}\left(Q_{1}, Q_{2}\right)\right. \\
& \left.\quad+\left(1-\tilde{\beta}_{H}\right) \bar{A}_{1}^{H}\left(Q_{1}, R_{2}\right)\right]=0, \\
& \tilde{\beta}_{L}\left(Q_{2}-\pi_{2}^{L}\right)+\tilde{\beta}_{H} \Gamma_{1} \tilde{\rho}_{2}\left(Q_{1}, Q_{2}\right)\left(Q_{2}-\pi_{2}^{H}\right)=0 \\
& \left(1-\tilde{\beta}_{L}\right)\left(R_{2}-\pi_{1}^{L} \pi_{2}^{L}\right)+\left(1-\tilde{\beta}_{H}\right) \Gamma_{0} \bar{\rho}_{2}\left(Q_{1}, R_{2}\right)\left(R_{2}-\pi_{1}^{H} \pi_{2}^{H}\right)=0,
\end{aligned}
$$

and by the relation

$$
Q_{1} Q_{2}=R_{2}
$$

\footnotetext{
${ }^{14}$ We are grateful to an anonymous referee for bringing this point to our attention.
} 
As already mentioned only one price for the first-period contract can exist. $\tilde{A}_{1}^{i}$ and $\bar{A}_{1}^{i}$ indicate demand for this contract by the groups which choose the sequential and the simultaneous second-period contract, resp., and $\tilde{\rho}_{2}, \bar{\rho}_{2}$ represent the demand ratios for the latter contracts.

One finds indeed that in case of logarithmic utility and for any given values of $\pi_{1}^{i}, \pi_{2}^{i}, i=L, H$, and $\gamma_{0}$, a ratio of the group shares $\tilde{\beta}_{H} / \tilde{\beta}_{L} \neq 1$ exists such that (3.12)-(3.15) are fulfilled with positive prices $Q_{1}, Q_{2}, R_{2}$.

Note that in case of $\tilde{\beta}_{H}=\tilde{\beta}_{L}$ the system (3.12)-(3.15) cannot be fulfilled in general: The reason is that in this case the whole population can be thought of as being split into two parts - each with the same ratio of high and low-risk individuals equal to $\gamma_{0} /\left(1-\gamma_{0}\right)$. If these two parts were completely separated, they would conform with the two regimes - the sequential and the simultaneous-discussed in 3.2, and prices $\left(\tilde{Q}_{1}, \tilde{Q}_{2}\right),\left(\bar{Q}_{1}, \bar{R}_{2}\right)$ with $\tilde{Q}_{1}>\bar{Q}_{1}$ and $\tilde{Q}_{1} \tilde{Q}_{2}<\bar{R}_{2}$ would arise. However, in the allmarkets-open situation only one price $Q_{1}$ for the first-period contract can prevail, and it is straightforward to see that-under mild conditions concerning the influence of the prices on the composition of demand (similar to those of Lemma 9)-this price must lie between $\tilde{Q}_{1}$ and $\bar{Q}_{1}$ and that, moreover, the relation $Q_{1} Q_{2}<R_{2}$ follows from (3.12)-(3.14), which conflicts with (3.15). ${ }^{15}$

However, in case of $\tilde{\beta}_{H} \neq \tilde{\beta}_{L}$, the zero-profit prices of the second-period contracts in the all-markets-open regime differ from those in the separate regimes, which makes it possible that some ratio $\tilde{\beta}_{H} / \tilde{\beta}_{L}$ exists, such that $(3.15)$ is fulfilled. Obviously, this situation can only occur, if insurance companies correctly anticipate the ratio $\tilde{\beta}_{H} / \tilde{\beta}_{L}$ and offer the contracts at corresponding zero-profit prices. As a next step, we extend the equilibrium concept of Section 3.3 accordingly: We call a set, consisting of an $A_{1^{-}}, A_{2^{-}}, D_{2}$-contract with prices $Q_{1}, Q_{2}, R_{2}$, an all-markets-open equilibrium, (i) if prices are such that individuals are indifferent between choosing the simultaneous and the sequential second-period contract, i.e. $Q_{1} Q_{2}=R_{2}$, (ii) if these prices together with appropriate shares $\tilde{\beta}_{i},\left(1-\tilde{\beta}_{i}\right), i=L, H$, describing the proportions at which individuals choose sequential and simultaneous second-period contracts, resp., produce zero expected profits, and (iii) if no other contract exists, which is preferred by at least some individuals and which allows a nonnegative profit.

It turns out that no such equilibrium exists. To see this, assume that a situation described by $(3.12)-(3.15)$ with $\tilde{\beta}_{H} / \tilde{\beta}_{L} \neq 1$ prevails. One can always find another contract which is preferred by at least one type of individuals and which allows a nonnegative profit: Consider the case $\tilde{\beta}_{H}>\tilde{\beta}_{L}$, which means that a higher share $\gamma_{0} \tilde{\beta}_{H} /\left(\gamma_{0} \tilde{\beta}_{H}+\left(1-\gamma_{0}\right) \tilde{\beta}_{L}\right)>\gamma_{0}$ of type- $H$ individuals than of type- $L$ individuals chooses the sequential second-period contract in the all-markets-open regime. This implies a higher extent of adverse selection and hence a higher zero-profit price of that contract, compared to the case that all individuals would choose the sequential second-period contract. Therefore, by offering the sequential second-period contract at a lower price than the prevailing zero-profit price, an insurance company induces all individuals (of both types) to buy that contract (as then $Q_{1} Q_{2}<R_{2}$ ) and makes a

\footnotetext{
${ }^{15}$ As an illustration, remember that for logarithmic utility the cross-price effect of $Q_{1}$ on the demand-ratios $\tilde{\rho}_{2}$ and $\bar{\rho}_{2}$ is zero. Therefore, $\tilde{Q}_{2}, \bar{R}_{2}$ also arise as zero-profit prices in the all-markets-open situation and the above price relations are immediate.
} 
nonnegative profit. Equivalent considerations apply for the case $\tilde{\beta}_{H}<\tilde{\beta}_{L}$, where firms have an incentive to offer the simultaneous second-period contract at a lower price in order to attract both groups of individuals. This explains why an all-markets-open regime cannot represent an equilibrium. Instead only an equilibrium with sequential contracts can prevail, as discussed in Section 3.3.

\section{Concluding remarks}

Provision for old age can be made through a variety of annuity products, which differ in the terms concerning asset accumulation and the payout path. In the present paper we have concentrated on annuities which run over a limited time only and have to be supplemented by a second contract. This additional contract can either be bought simultaneously with the first or later, when an individual knows that she has survived some years of retirement. We have characterised demand, given these two possibilities, and we have studied the consequences of the adverse-selection phenomenon in this market. It turns out that only a situation, where all individuals demand sequential contracts represents an equilibrium. This is favourable for the high-risk group, while the low-risk group would be better off with the simultaneous regime. This result shows some similarity to conclusions from other models with asymmetric information, where typically the low-risk groups do not receive their first-best contract.

Ultimately, the important relations which drive the results are $\tilde{Q}_{1}>\bar{Q}_{1}$ and $\tilde{Q}_{1} \tilde{Q}_{2}<\bar{R}_{2}$, reflecting the differing extent of adverse selection for the sequential and simultaneous annuity contracts: The reason is that in the sequential regime, provision for period 2 goes via period 1 where the high-risk individuals are in a common pool with the low-risk individuals, who put more weight on the first period of retirement than on the second. In the simultaneous regime, however, demand for each single-period contract satisfies only the need for future consumption in the respective retirement period. This explains why in the sequential regime the problem of adverse selection for first-period provision is more severe (hence $\tilde{Q}_{1}>\bar{Q}_{1}$ ), while it is relieved for period-two provision (hence $\tilde{Q}_{1} \tilde{Q}_{2}<\bar{R}_{2}$ ).

One important assumption was used in this study in order to derive the results: insurance companies can credibly commit to offer an insurance contract at a fixed price one period later. Without commitment, one would have to introduce some way of how beliefs concerning the expected price of a future contract are formulated. At first glance, in such a formulation the occurrence of uncertainty would make future contracts less attractive, and, as a consequence, the sequential regime less likely to represent an equilibrium.

However, price uncertainty is not the only variation of the model, which works in favour of the simultaneous contracts. They also become more attractive, if one considers a scenario, related to that of Brugiavini [1993], where individuals learn about their life expectancy as they age. In our model this means that in the working period the individuals (and the firms) do not know their risk types. However this information is revealed to them (and only to them) in the first retirement period, when the sequential second-period contract can be bought. Hence, the latter contract continues to be affected by the adverse selection problem, while this is no longer the case for the 
period-one contract and the simultaneous period-two contract. As a consequence of this setting, the period-one contract and the simultaneous period-two contract are offered at prices based on the average survival probabilities (viz. $\hat{Q}_{1} \equiv \gamma_{0} \pi_{1}^{H}+\left(1-\gamma_{0}\right) \pi_{1}^{L}$ and $\left.\hat{R}_{2} \equiv \gamma_{0} \pi_{1}^{H} \pi_{2}^{H}+\left(1-\gamma_{0}\right) \pi_{1}^{L} \pi_{2}^{L}\right)$, while the sequential period-two contract is, due to adverse selection, offered at a price $Q_{2}$ larger than $\hat{R}_{2} / \hat{Q}_{1}$. Then all individuals will provide for the second retirement period by the purchase of the simultaneous contract in the working period 0 , and leave it open until period 1 , whether to adapt their consumption path over retirement according to their longevity risk, which by then they will know, via the purchase of the $A_{2}$-contract. Moreover, as in Brugiavini [1993], it turns out that no trade takes place in period 1 . The reason is that by choosing the appropriate behaviour, the individuals reveal their types: only high-risk individuals want to buy additional insurance ( $A_{2}$-annuities), while low-risk individuals would like to reduce provision for period 2 , by taking up a loan, if the capital market allows this. In any case, one can show that with individually fair prices-as a result of type-revelation-for the $A_{2}$-contract (or for appropriate loans), the individuals cannot improve their welfare by adapting provision for period 2 . Altogether, we conclude that the elimination of asymmetric information in the working period makes the sequential second-period contract useless and only the simultaneous contracts are traded in equilibrium. $^{16}$

The main conclusion from our contribution is that adverse selection has more severe consequences on the annuity market than recognised in studies using the standard overlapping-generations model. These mainly concentrate on the influence of adverse selection on a single rate of return for a uniform period of retirement. By extending this model and making the realistic assumption that provision for retirement need not be made through a once-and-for-all annuity contract, but can be made through different contracts for earlier and later phases of retirement, one finds that adverse selection also affects the choice of contracts as well as the existence and properties of equilibria.

It is well-known that the observed data from the private annuity market reveal puzzling peculiarities. The most frequently mentioned is that people buy considerably less annuity contracts than one would expect, given their higher return compared to that on other forms of wealth [see, e.g., Friedman and Warshawsky, 1990]. Another one is the wide-spread use of so-called "years certain" contracts, ${ }^{17}$ which is also difficult to explain within a standard model of household decision. Given the result of our analysis that using time-limited contracts in the sequential regime represents an equilibrium, the observed fact, mentioned in the introduction, that time-limited contracts make up only a small share of all actually purchased annuities adds another puzzle: in fact the majority of individuals buy life annuities for the whole period of retirement altogether, which can be interpreted as the simultaneous regime in our model. Possible

\footnotetext{
16 This version of the model represents an example of dynamic adverse selection, where information is revealed over time to the participating individuals [for an overview see Bolton and Dewatripont, 2005, ch. 9]. Note, however, that in our framework of price competition for one-period contracts, the typical question of renegotiation does not arise.

17 These contracts offer guaranteed payouts for a fixed number periods (possibly to descendants), and then regular payouts until death. Obviously, their price is higher than that of annuities without a guaranteed payout phase.
} 
explanations could be seen in the lack of price commitment of insurance companies and in the lack of private information of the individuals in the working period to know their life expectancy, as discussed above. A further crucial issue of our analysis is the range of annuity contracts or their combinations available to the individuals. Further research is needed in order to clarify the functioning of the market, if additional types of contracts, for instance packages of first- and second-period insurance, are introduced.

\section{Appendix}

Proof of Lemma 1: By use of the Eqs. in (2.8a), (2.9) and (2.10), Eq. (2.6) can be written as $-Q_{1} Q_{2} u^{\prime}\left(w_{0}-Q_{1} A_{1}^{i}-R_{2} D_{2}^{i}\right)+\pi_{1}^{i} \partial \varphi^{i}\left(A_{1}^{i}, Q_{2}, D_{2}^{i}\right) / \partial D_{2}^{i}=0$, where the first term on the LHS in general will not be equal to the term $-R_{2} u^{\prime}\left(w_{0}-Q_{1} A_{1}^{i}-\right.$ $R_{2} D_{2}^{i}$ ) of the Eq. in (2.7a). As we know that (2.6) must always be fulfilled, this means that the equations in (2.7a) and (2.8a) cannot hold simultaneously. By the same reasoning, one observes that if (2.8a) holds, (2.7b) can be fulfilled only if $R_{2} \geq Q_{1} Q_{2}$, and analogously for (2.7a) and (2.8b).

Proof of Lemma 2: Part (i): We denote by $W^{i}$ the LHS of (2.6) and by $V^{i}$ the LHS of the equation in (2.8a), where $D_{2}^{i}=0$. Implicit differentiation gives

$$
\left(\begin{array}{ll}
\frac{\partial A_{1}^{i}}{\partial Q_{1}} & \frac{\partial A_{1}^{i}}{\partial Q_{2}} \\
\frac{\partial A_{2}^{i}}{\partial Q_{1}} & \frac{\partial A_{2}^{i}}{\partial Q_{2}}
\end{array}\right)=-\left(\begin{array}{ll}
\frac{\partial W^{i}}{\partial A_{1}^{i}} & \frac{\partial W^{i}}{\partial A_{2}^{i}} \\
\frac{\partial V^{i}}{\partial A_{1}^{i}} & \frac{\partial V^{i}}{\partial A_{2}^{i}}
\end{array}\right)^{-1}\left(\begin{array}{ll}
\frac{\partial W^{i}}{\partial Q_{1}} & \frac{\partial W^{i}}{\partial Q_{2}} \\
\frac{\partial V^{i}}{\partial Q_{1}} & \frac{\partial V^{i}}{\partial Q_{2}}
\end{array}\right)
$$

We find that

$$
\begin{aligned}
& \frac{\partial V^{i}}{\partial A_{1}^{i}}=-Q_{2} u^{\prime \prime}\left(c_{1}^{i}\right)>0, \\
& \frac{\partial V^{i}}{\partial A_{2}^{i}}=Q_{2}^{2} u^{\prime \prime}\left(c_{1}^{i}\right)+\pi_{2}^{i} u^{\prime \prime}\left(c_{2}^{i}\right)<0, \\
& \frac{\partial W^{i}}{\partial A_{1}^{i}}=Q_{1}^{2} u^{\prime \prime}\left(c_{0}^{i}\right)+\pi_{1}^{i} \frac{\partial^{2} \varphi\left(A_{1}^{i}, Q_{2}\right)}{\partial w_{1}^{i 2}}=Q_{1}^{2} u^{\prime \prime}\left(c_{0}^{i}\right)+\pi_{1}^{i}\left(1-Q_{2} \frac{\partial A_{2}^{i}\left(A_{1}^{i}, Q_{2}\right)}{\partial A_{1}^{i}}\right) .
\end{aligned}
$$

due to (2.9), where $A_{2}^{i}\left(A_{1}^{i}, Q_{2}\right)$ denotes annuity demand $A_{2}^{i}$ for fixed $W_{1}^{i}=A_{1}^{i}$, determined by (2.8a). Hence, $\partial A_{2}^{i}\left(A_{1}^{i}, Q_{2}\right) / \partial A_{1}^{i}$ is derived from implicit differentiation of (2.8a) as

$$
\frac{\partial A_{2}^{i}}{\partial A_{1}^{i}}=\frac{Q_{2} u^{\prime \prime}\left(c_{1}^{i}\right)}{\partial V^{i} / \partial A_{2}^{i}} .
$$


By use of (A3)-(A5) we obtain

$$
\frac{\partial W^{i}}{\partial A_{1}^{i}}=Q_{1}^{2} u^{\prime \prime}\left(c_{0}^{i}\right)+\pi_{1}^{i} \pi_{2}^{i} \frac{u^{\prime \prime}\left(c_{1}^{i}\right) u^{\prime \prime}\left(A_{2}^{i}\right)}{\partial V^{i} / \partial A_{2}^{i}}<0 .
$$

Further, we have:

$$
\begin{aligned}
& \frac{\partial W^{i}}{\partial A_{2}^{i}}=0 \\
& \frac{\partial V^{i}}{\partial Q_{1}}=0 \\
& \frac{\partial V^{i}}{\partial Q_{2}}=-u^{\prime}\left(c_{1}^{i}\right)+Q_{2} A_{2}^{i} u^{\prime \prime}\left(c_{1}^{i}\right)<0, \\
& \frac{\partial W^{i}}{\partial Q_{1}}=-u^{\prime}\left(c_{0}^{i}\right)+Q_{1} A_{1}^{i} u^{\prime \prime}\left(c_{0}^{i}\right)<0, \\
& \frac{\partial W^{i}}{\partial Q_{2}}=\pi_{1}^{i} \frac{\partial^{2} \varphi^{i}\left(A_{1}^{i}, Q_{2}\right)}{\partial A_{1}^{i} \partial Q_{2}}=-\pi_{1}^{i} u^{\prime \prime}\left(c_{1}^{i}\right)\left(A_{2}^{i}+Q_{2} \frac{\partial A_{2}^{i}\left(A_{1}^{i}, Q_{2}\right)}{\partial Q_{2}}\right),
\end{aligned}
$$

due to (2.9). By use of $\varepsilon_{2}^{i}\left(A_{1}^{i}, Q_{2}\right) \equiv \frac{\partial A_{2}^{i}\left(A_{1}^{i}, Q_{2}\right)}{\partial Q_{2}} \frac{Q_{2}}{A_{2}^{i}}$, denoting the price elasticity of demand $A_{2}^{i}\left(A_{1}^{i}, Q_{2}\right)$ for fixed $w_{1}^{i}$, together with (differentiate (2.8a) implicitly and use (A2) and (A3))

$$
\frac{\partial A_{2}^{i}\left(A_{1}^{i}, Q_{2}\right)}{\partial Q_{2}}=-\frac{\partial V^{i} / \partial Q_{2}}{\partial V^{i} / \partial A_{2}^{i}}<0
$$

we obtain

$\frac{\partial W^{i}}{\partial Q_{2}}=-\pi_{1}^{i} A_{2}^{i} u^{\prime \prime}\left(c_{1}^{i}\right)\left(1+\varepsilon_{2}^{i}\left(A_{1}^{i}, Q_{2}\right)\right) \underset{<}{\gtrless} 0 \Leftrightarrow \varepsilon_{2}^{i}\left(A_{1}^{i}, Q_{2}\right) \underset{<}{<}-1, \varepsilon_{2}^{i}\left(A_{1}^{i}, Q_{2}\right)<0$.

Now let

$$
N \equiv \underbrace{\frac{\partial W^{i}}{\partial A_{1}^{i}}}_{<0} \underbrace{\frac{\partial V^{i}}{\partial A_{2}^{i}}}_{<0}-\underbrace{\frac{\partial W^{i}}{\partial A_{2}^{i}} \frac{\partial V^{i}}{\partial A_{1}^{i}}}_{=0}>0
$$

Inverting the first matrix on the RHS of (A1) and multiplying gives (note the inequalities from above):

$$
\frac{\partial A_{1}^{i}\left(Q_{1}, Q_{2}\right)}{\partial Q_{1}}=-\frac{1}{N}(\underbrace{\frac{\partial V^{i}}{\partial A_{2}^{i}}}_{<0} \underbrace{\frac{\partial W^{i}}{\partial Q_{1}}}_{<0}-\underbrace{\frac{\partial W^{i}}{\partial A_{2}^{i}} \frac{\partial V^{i}}{\partial Q_{1}}}_{=0})<0,
$$




$$
\begin{aligned}
& \frac{\partial A_{2}^{i}\left(Q_{1}, Q_{2}\right)}{\partial Q_{1}}=-\frac{1}{N}(-\underbrace{\frac{\partial V^{i}}{\partial A_{1}^{i}}}_{>0} \underbrace{\frac{\partial W^{i}}{\partial Q_{1}}}_{<0}+\underbrace{\frac{\partial W^{i}}{\partial A_{1}^{i}} \frac{\partial V^{i}}{\partial Q_{1}}}_{=0})<0, \\
& \frac{\partial A_{1}^{i}\left(Q_{1}, Q_{2}\right)}{\partial Q_{2}}=-\frac{1}{N}(\underbrace{\frac{\partial V^{i}}{\partial A_{2}^{i}} \frac{\partial W^{i}}{\partial Q_{2}}}_{<0}-\underbrace{\frac{\partial W^{i}}{\partial A_{2}^{i}} \frac{\partial V^{i}}{\partial Q_{2}}}_{=0}) \geq 0 \Leftrightarrow \varepsilon_{2}^{i}\left(A_{1}^{i}, Q_{2}\right) \geq-1, \\
& \frac{\partial A_{2}^{i}\left(Q_{1}, Q_{2}\right)}{\partial Q_{2}}=-\frac{1}{N}(-\underbrace{\frac{\partial V^{i}}{\partial A_{1}^{i}}}_{>0} \frac{\partial W^{i}}{\partial Q_{2}}+\underbrace{\frac{\partial W^{i}}{\partial A_{1}^{i}}}_{<0} \underbrace{\frac{\partial V^{i}}{\partial Q_{2}}}_{<0}),
\end{aligned}
$$

which can rearranged to [use (A2), (A6), (A9), (A11) and (A12)]

$$
\begin{aligned}
\frac{\partial A_{2}^{i}\left(Q_{1}, Q_{2}\right)}{\partial Q_{2}}= & -\frac{1}{N}\left[Q_{2} u^{\prime \prime}\left(c_{1}^{i}\right)\left(\frac{\partial W^{i}}{\partial Q_{2}}+A_{2}^{i} \frac{\partial W^{i}}{\partial A_{1}^{i}}\right)-\frac{\partial W^{i}}{\partial A_{1}^{i}} u^{\prime}\left(c_{1}^{i}\right)\right] \\
= & -\frac{1}{N}\left[Q_{2} u^{\prime \prime}\left(c_{1}^{i}\right)\left(-\frac{\pi_{1}^{i} Q_{2} u^{\prime \prime}\left(c_{1}^{i}\right) u^{\prime}\left(c_{1}^{i}\right)}{\partial V^{i} / \partial A_{2}^{i}}+Q_{1}^{2} A_{2}^{i} u^{\prime \prime}\left(c_{0}^{i}\right)\right)\right. \\
& \left.-\frac{\partial W^{i}}{\partial A_{1}^{i}} u^{\prime}\left(c_{1}^{i}\right)\right]<0 .
\end{aligned}
$$

Part (ii): Let now denote $W^{i}$ and $V^{i}$ the LHS's of (2.6) and (2.7a), resp. With $A_{2}^{i}=0$ these equations reduce to (see (2.9), (2.10))

$$
\begin{aligned}
& -Q_{1} u^{\prime}\left(w_{0}-Q_{1} A_{1}^{i}-R_{2} D_{2}^{i}\right)+\pi_{1}^{i} u^{\prime}\left(A_{1}^{i}\right)=0, \\
& -R_{2} u^{\prime}\left(w_{0}-Q_{1} A_{1}^{i}-R_{2} D_{2}^{i}\right)+\pi_{1}^{i} \pi_{2}^{i} u^{\prime}\left(D_{2}^{i}\right)=0 .
\end{aligned}
$$

The formula for implicit differentiation of these equations is the same as (A1), when $A_{2}^{i}$ is replaced by $D_{2}^{i}$ and $Q_{2}$ by $R_{2}$.

We find that

$$
\begin{array}{ll}
\frac{\partial W^{i}}{\partial A_{1}^{i}}=Q_{1}^{2} u^{\prime \prime}\left(c_{0}^{i}\right)+\pi_{1}^{i} u^{\prime \prime}\left(c_{1}^{i}\right)<0, & \frac{\partial W^{i}}{\partial D_{2}^{i}}=Q_{1} R_{2} u^{\prime \prime}\left(c_{0}^{i}\right)<0, \\
\frac{\partial V^{i}}{\partial A_{1}^{i}}=Q_{1} R_{2} u^{\prime \prime}\left(c_{0}^{i}\right)<0, & \frac{\partial V^{i}}{\partial D_{2}^{i}}=R_{2}^{2} u^{\prime \prime}\left(c_{0}^{i}\right)+\pi_{1}^{i} \pi_{2}^{i} u^{\prime \prime}\left(c_{2}^{i}\right)<0, \\
\frac{\partial W^{i}}{\partial Q_{1}}=-u^{\prime}\left(c_{0}^{i}\right)+Q_{1} A_{1}^{i} u^{\prime \prime}\left(c_{0}^{i}\right)<0, & \frac{\partial W^{i}}{\partial R_{2}}=Q_{1} D_{2} u^{\prime \prime}\left(c_{0}^{i}\right)<0, \\
\frac{\partial V^{i}}{\partial Q_{1}}=R_{2} A_{1}^{i} u^{\prime \prime}\left(c_{0}^{i}\right)<0, & \frac{\partial V^{i}}{\partial R_{2}}=-u^{\prime}\left(c_{0}^{i}\right)+R_{2} D_{2}^{i} u^{\prime \prime}\left(c_{0}^{i}\right)<0 .
\end{array}
$$


Now let

$$
M \equiv \frac{\partial W^{i}}{\partial A_{1}^{i}} \frac{\partial V^{i}}{\partial D_{2}^{i}}-\frac{\partial W^{i}}{\partial D_{2}^{i}} \frac{\partial V^{i}}{\partial A_{1}^{i}}>0,
$$

where the positive sign follows immediately from easy calculation by use of (A22) and (A23). One derives immediately, using (A15)-(A19), together with (A22)-(A26) and by some straightforward computations:

$$
\begin{gathered}
\frac{\partial A_{1}^{i}\left(Q_{1}, R_{2}\right)}{\partial Q_{1}}=-\frac{1}{M}\left(\frac{\partial V^{i}}{\partial D_{2}^{i}} \frac{\partial W^{i}}{\partial Q_{1}}-\frac{\partial W^{i}}{\partial D_{2}^{i}} \frac{\partial V^{i}}{\partial Q_{1}}\right)<0, \\
\frac{\partial D_{2}^{i}\left(Q_{1}, R_{2}\right)}{\partial R_{2}}=-\frac{1}{M}\left(-\frac{\partial V^{i}}{\partial A_{1}^{i}} \frac{\partial W^{i}}{\partial R_{2}}+\frac{\partial W^{i}}{\partial A_{1}^{i}} \frac{\partial V^{i}}{\partial R_{2}}\right)<0 .
\end{gathered}
$$

Further, we find that

$$
\begin{aligned}
\frac{\partial D_{2}^{i}\left(Q_{1}, R_{2}\right)}{\partial Q_{1}} & =-\frac{1}{M}\left(-\frac{\partial V^{i}}{\partial A_{1}^{i}} \frac{\partial W^{i}}{\partial Q_{1}}+\frac{\partial W^{i}}{\partial A_{1}^{i}} \frac{\partial V^{i}}{\partial Q_{1}}\right) \\
& =-\frac{1}{M} R_{2} u^{\prime \prime}\left(c_{0}^{i}\right)\left(-Q_{1} \frac{\partial W^{i}}{\partial Q_{1}}+A_{1}^{i} \frac{\partial W^{i}}{\partial A_{1}^{i}}\right) .
\end{aligned}
$$

Substituting the price elasticity of demand $A_{1}^{i}\left(D_{2}^{i}, Q_{1}\right)$ for fixed $D_{2}^{i}$, defined as $\eta_{1}^{i} \equiv$ $\frac{\partial A_{1}^{i}\left(D_{2}^{i}, Q_{1}\right)}{\partial Q_{1}} \frac{Q_{1}}{A_{1}^{i}}$, together with $\frac{\partial A_{1}^{i}\left(D_{2}^{i}, Q_{1}\right)}{\partial Q_{1}}=-\frac{\partial W^{i} / \partial Q_{1}}{\partial W^{i} / \partial A_{1}^{i}}<0$ (differentiate (A20) implicitly) into (A29) yields

$$
\frac{\partial D_{2}^{i}\left(Q_{1}, R_{2}\right)}{\partial Q_{1}}=-\frac{1}{M} R_{2} u^{\prime \prime}\left(c_{0}^{i}\right) A_{1}^{i} \frac{\partial W^{i}}{\partial A_{1}^{i}}\left(\eta_{1}+1\right) \underset{<}{\gtrless} 0 \Leftrightarrow \eta_{1}^{i} \geq-1, \quad \eta_{1}^{i}<0 .
$$

Analogously, we use the price elasticity $\eta_{2}^{i} \equiv \frac{\partial D_{2}^{i}\left(A_{1}^{i}, R_{2}\right)}{\partial R_{2}} \frac{R_{2}}{D_{2}^{i}}$ of demand $D_{2}^{i}\left(A_{1}^{i}, R_{2}\right)$ for fixed $A_{1}^{i}$, together with $\frac{\partial D_{2}^{i}\left(A_{1}^{i}, R_{2}\right)}{\partial R_{2}}=-\frac{\partial V^{i} / \partial R_{2}}{\partial V^{i} / \partial D_{2}^{i}}<0$ (differentiate (A21) implicitly) to determine the sign of $\partial A_{1}^{i}\left(Q_{1}, R_{2}\right) / \partial R_{2}$ :

$$
\begin{aligned}
\frac{\partial A_{1}^{i}\left(Q_{1}, R_{2}\right)}{\partial R_{2}} & =-\frac{1}{M}\left(\frac{\partial V^{i}}{\partial D_{2}^{i}} \frac{\partial W^{i}}{\partial R_{2}}-\frac{\partial W^{i}}{\partial D_{2}^{i}} \frac{\partial V^{i}}{\partial R_{2}}\right) \\
& =-\frac{1}{M} Q_{1} u^{\prime \prime}\left(c_{0}^{i}\right)\left(D_{2}^{i} \frac{\partial V^{i}}{\partial D_{2}^{i}}-R_{2} \frac{\partial V^{i}}{\partial R_{2}}\right) \\
& =-\frac{1}{M} Q_{1} u^{\prime \prime}\left(c_{0}^{i}\right) D_{2}^{i} \frac{\partial V^{i}}{\partial D_{2}^{i}}\left(1+\eta_{2}^{i}\right) \underset{<}{\gtrless} 0 \Leftrightarrow \eta_{2}^{i} \geq-1, \quad \eta_{2}^{i}<0 .
\end{aligned}
$$


Proof of Lemma 3: Part (i): We define $W^{i}$ and $V^{i}$ as in the proof of Lemma 2, part (i). The formula for implicit differentiation of (2.6) and the Eq. in (2.8a) is the same as in (A1), where $Q_{1}, Q_{2}$ are replaced by $\pi_{1}^{i}, \pi_{2}^{i}$, resp.

We find that

$$
\begin{aligned}
& \frac{\partial W^{i}}{\partial \pi_{1}^{i}}=\frac{\partial \varphi}{\partial w_{1}^{i}}>0, \\
& \frac{\partial W^{i}}{\partial \pi_{2}^{i}}=\pi_{1}^{i} \frac{\partial^{2} \varphi^{i}\left(w_{1}^{i}, Q_{2}\right)}{\partial w_{1}^{i} \partial \pi_{2}^{i}}=-\pi_{1}^{i} Q_{2} u^{\prime \prime}\left(c_{1}^{i}\right) \frac{\partial A_{2}^{i}\left(w_{1}^{i}, Q_{2}\right)}{\partial \pi_{2}}=\frac{\pi_{1}^{i} Q_{2} u^{\prime \prime}\left(c_{1}^{i}\right) u^{\prime}\left(c_{2}^{i}\right)}{Q_{2}^{2} u^{\prime \prime}\left(c_{1}^{i}\right)+\pi_{2} u^{\prime \prime}\left(c_{2}^{i}\right)}>0,
\end{aligned}
$$

due to (2.9), where $\partial A_{2}^{i}\left(w_{1}^{i}, Q_{2}\right) / \partial \pi_{2}$ (denoting the change of annuity demand for fixed $w_{1}^{i}$, if $\pi_{2}^{i}$ increases), is determined by implicit differentiation of the equation in (2.8a).

Furthermore:

$$
\frac{\partial V^{i}}{\partial \pi_{1}^{i}}=0, \quad \frac{\partial V^{i}}{\partial \pi_{2}^{i}}=u^{\prime}\left(c_{2}^{i}\right)>0 .
$$

Using these computations (A31)-(A33), together with (A2), (A3), (A6), (A7) and (A14) in (A15)-(A18) gives

$$
\begin{aligned}
& \frac{\partial A_{1}^{i}\left(Q_{1}, Q_{2}\right)}{\partial \pi_{1}^{i}}=-\frac{1}{N} \frac{\partial V^{i}}{\partial A_{2}^{i}} \frac{\partial W^{i}}{\partial \pi_{1}^{i}}>0, \\
& \frac{\partial A_{2}^{i}\left(Q_{1}, Q_{2}\right)}{\partial \pi_{1}^{i}}=\frac{1}{N} \frac{\partial V^{i}}{\partial A_{1}^{i}} \frac{\partial W^{i}}{\partial \pi_{1}^{i}}>0, \\
& \frac{\partial A_{1}^{i}\left(Q_{1}, Q_{2}\right)}{\partial \pi_{2}^{i}}=-\frac{1}{N} \frac{\partial V^{i}}{\partial A_{2}^{i}} \frac{\partial W^{i}}{\partial \pi_{2}^{i}}>0, \\
& \frac{\partial A_{2}^{i}\left(Q_{1}, Q_{2}\right)}{\partial \pi_{2}^{i}}=-\frac{1}{N}\left(-\frac{\partial V^{i}}{\partial A_{1}^{i}} \frac{\partial W^{i}}{\partial \pi_{2}^{i}}+\frac{\partial W^{i}}{\partial A_{1}^{i}} \frac{\partial V^{i}}{\partial \pi_{2}^{i}}\right)>0 .
\end{aligned}
$$

Part (ii): We define $W^{i}$ and $V^{i}$ as in part (ii) of Lemma 2 and use the same formula (A1) for implicit differentiation of (A20), (A21), where $A_{2}^{i}, Q_{1}, Q_{2}$ are replaced by $D_{2}^{i}, \pi_{1}^{i}, \pi_{2}^{i}$ resp.

We find that

$$
\begin{array}{ll}
\frac{\partial W^{i}}{\partial \pi_{1}^{i}}=u^{\prime}\left(c_{1}^{i}\right)>0, & \frac{\partial W^{i}}{\partial \pi_{2}^{i}}=0, \\
\frac{\partial V^{i}}{\partial \pi_{1}^{i}}=\pi_{2}^{i} u^{\prime}\left(c_{2}^{i}\right)>0, & \frac{\partial V^{i}}{\partial \pi_{2}^{i}}=\pi_{1}^{i} u^{\prime}\left(c_{2}^{i}\right)>0 .
\end{array}
$$


Using these computations (A34) and (A35), together with (A22), (A24) and (A26), in (A27)-(A30), it follows [note that $R_{2} u^{\prime}\left(c_{1}^{i}\right)=\pi_{2}^{i} Q_{1} u^{\prime}\left(c_{2}^{i}\right)$ due to (A20) and (A21)]:

$$
\begin{aligned}
& \frac{\partial A_{1}^{i}\left(Q_{1}, R_{2}\right)}{\partial \pi_{1}^{i}}=-\frac{1}{M}\left(\frac{\partial V^{i}}{\partial D_{2}^{i}} \frac{\partial W^{i}}{\partial \pi_{1}^{i}}-\frac{\partial W^{i}}{\partial D_{2}^{i}} \frac{\partial V^{i}}{\partial \pi_{1}^{i}}\right)=-\frac{1}{M} \pi_{1}^{i} \pi_{2}^{i} u^{\prime \prime}\left(c_{0}^{i}\right) u^{\prime}\left(c_{1}^{i}\right)>0, \\
& \frac{\partial D_{2}^{i}\left(Q_{1}, R_{2}\right)}{\partial \pi_{1}^{i}}=-\frac{1}{M}\left(-\frac{\partial V^{i}}{\partial A_{1}^{i}} \frac{\partial W^{i}}{\partial \pi_{1}^{i}}+\frac{\partial W^{i}}{\partial A_{1}^{i}} \frac{\partial V^{i}}{\partial \pi_{1}^{i}}\right)=-\frac{1}{M} \pi_{1}^{i} u^{\prime \prime}\left(c_{1}^{i}\right) \pi_{2}^{i} u^{\prime}\left(c_{2}^{i}\right)>0, \\
& \frac{\partial A_{1}^{i}\left(Q_{1}, R_{2}\right)}{\partial \pi_{2}^{i}}=\frac{1}{M} \frac{\partial W^{i}}{\partial D_{2}^{i}} \frac{\partial V^{i}}{\partial \pi_{2}^{i}}<0, \\
& \frac{\partial D_{1}^{i}\left(Q_{1}, R_{2}\right)}{\partial \pi_{2}^{i}}=-\frac{1}{M} \frac{\partial W^{i}}{\partial A_{1}^{i}} \frac{\partial V^{i}}{\partial \pi_{2}^{i}}>0 .
\end{aligned}
$$

Proof of Lemma 7: As a preparation, we calculate the demand functions in either regime: The conditions (2.6) and (2.8a) together with (2.9) determine annuity demand $\tilde{A}_{1}^{i}$ and $\tilde{A}_{2}^{i}$ in the sequential regime. For CRRA utility (3.2) one computes

$$
\tilde{A}_{1}^{i}=\left(1+\pi_{2}^{i 1 / \sigma} Q_{2}^{1-1 / \sigma}\right) \frac{\pi_{1}^{i 1 / \sigma}}{Q_{1}^{1 / \sigma}} \frac{w_{0}}{X^{i}}, \quad \tilde{A}_{2}^{i}=\frac{\left(\pi_{1}^{i} \pi_{2}^{i}\right)^{1 / \sigma}}{\left(Q_{1} Q_{2}\right)^{1 / \sigma}} \frac{w_{0}}{X^{i}} .
$$

where $X^{i} \equiv 1+\pi_{1}^{i 1 / \sigma} Q_{1}^{1-1 / \sigma}+\left(\pi_{1}^{i} \pi_{2}^{i}\right)^{1 / \sigma}\left(Q_{1} Q_{2}\right)^{1-1 / \sigma}$. By use of (A20), (A21) in the Appendix and (3.2) we obtain annuity demand $\bar{A}_{1}^{i}$ and $\bar{D}_{2}^{i}$ in the simultaneous regime as

$$
\bar{A}_{1}^{i}=\frac{\pi_{1}^{i 1 / \sigma}}{Q_{1}^{1 / \sigma}} \frac{w_{0}}{Y^{i}}, \quad \bar{D}_{2}^{i}=\frac{\left(\pi_{1}^{i} \pi_{2}\right)^{i 1 / \sigma}}{R_{2}^{1 / \sigma}} \frac{w_{0}}{Y^{i}} .
$$

where $Y^{i} \equiv 1+\pi_{1}^{i 1 / \sigma} Q_{1}^{1-1 / \sigma}+\left(\pi_{1}^{i} \pi_{2}^{i}\right)^{1 / \sigma} R_{2}^{1-1 / \sigma}$.

For any $Q_{1}, Q_{2}$ and $R_{2}$ such that $Q_{1} Q_{2}=R_{2}$, we have $X^{i}=Y^{i}$. By use of this equality, together with the definitions of $\tilde{\rho}_{1}\left(Q_{1}, Q_{2}\right), \bar{\rho}_{1}\left(Q_{1}, R_{2}\right)$ and the first equation in (A36) and in (A37), $\tilde{\rho}_{1}\left(Q_{1}, Q_{2}\right)$ can be written as

$$
\tilde{\rho}_{1}\left(Q_{1}, Q_{2}\right)=\frac{1+\pi_{2}^{H 1 / \sigma} Q_{2}^{1-1 / \sigma}}{1+\pi_{2}^{L 1 / \sigma} Q_{2}^{1-1 / \sigma}} \bar{\rho}_{1}\left(Q_{1}, R_{2}\right)
$$

which is larger than $\bar{\rho}_{1}$, as $\pi_{2}^{H}>\pi_{2}^{L}$.

Proof of Lemma 8: For any $Q_{1}, Q_{2}$ and $R_{2}$ such that $Q_{1} Q_{2}=R_{2}$, the consumption level in the working period is the same in the sequential and in the simultaneous regime (compare the Proof of Lemma 4). Hence, $w_{0}-Q_{1} \tilde{A}_{1}^{i}\left(Q_{1}, Q_{2}\right)=$ $w_{0}-Q_{1} \bar{A}_{1}^{i}\left(Q_{1}, R_{2}\right)-R_{2} \bar{D}_{2}^{i}\left(Q_{1}, R_{2}\right)$ for $i=L, H$, which can be rearranged to (use Springer 
$\left.Q_{1} Q_{2}=R_{2}\right)$

$$
\bar{D}_{2}^{i}\left(Q_{1}, R_{2}\right)=\left(\tilde{A}_{1}^{i}\left(Q_{1}, Q_{2}\right)-\bar{A}_{1}^{i}\left(Q_{1}, R_{2}\right)\right) / Q_{2}
$$

By use of (A39) for $i=L, H$ and the definitions of $\bar{\rho}_{2}\left(Q_{1}, R_{2}\right), \tilde{\rho}_{1}\left(Q_{1}, Q_{2}\right)$, the difference $\tilde{\rho}_{1}\left(Q_{1}, Q_{2}\right)-\bar{\rho}_{2}\left(Q_{1}, R_{2}\right)$ can be written as

$$
\tilde{\rho}_{1}\left(Q_{1}, Q_{2}\right)-\bar{\rho}_{2}\left(Q_{1}, R_{2}\right)=\frac{\tilde{A}_{1}^{H}\left(Q_{1}, Q_{2}\right)}{\tilde{A}_{1}^{L}\left(Q_{1}, Q_{2}\right)}-\frac{\tilde{A}_{1}^{H}\left(Q_{1}, Q_{2}\right)-\bar{A}_{1}^{H}\left(Q_{1}, R_{2}\right)}{\tilde{A}_{1}^{L}\left(Q_{1}, Q_{2}\right)-\bar{A}_{1}^{L}\left(Q_{1}, R_{2}\right)},
$$

and further, by some easy transformations (use (A39) again),

$$
\tilde{\rho}_{1}\left(Q_{1}, Q_{2}\right)-\bar{\rho}_{2}\left(Q_{1}, R_{2}\right)=\frac{\bar{A}_{1}^{L}\left(Q_{1}, R_{2}\right)}{Q_{2} \bar{D}_{2}^{i}\left(Q_{1}, R_{2}\right)}\left(\frac{\bar{A}_{1}^{H}\left(Q_{1}, R_{2}\right)}{\bar{A}_{1}^{L}\left(Q_{1}, R_{2}\right)}-\frac{\tilde{A}_{1}^{H}\left(Q_{1}, Q_{2}\right)}{\tilde{A}_{1}^{L}\left(Q_{1}, Q_{2}\right)}\right) .
$$

From (A41) it follows that $\tilde{\rho}_{1}\left(Q_{1}, Q_{2}\right)<\bar{\rho}_{2}\left(Q_{1}, R_{2}\right)$, iff $\tilde{\rho}_{1}\left(Q_{1}, Q_{2}\right)>\bar{\rho}_{1}$ $\left(Q_{1}, R_{2}\right)$.

Proof of Lemma 9: Let $\tilde{Q}_{1}, \tilde{Q}_{2}$ be implicitly defined by the conditions (3.3) and (3.5) in the sequential regime and let $\tilde{R}_{2} \equiv \tilde{Q}_{1} \tilde{Q}_{2}$. Further, let $\bar{P}_{t}\left(Q_{1}, R_{2}\right), t=1,2$, be evaluated at $Q_{1}=\tilde{Q}_{1}, R_{2}=\tilde{R}_{2}$. First, we substitute (3.3) with $Q_{1}=\tilde{Q}_{1}, Q_{2}=\tilde{Q}_{2}$ into $\bar{P}_{1}\left(\tilde{Q}_{1}, \tilde{R}_{2}\right)$ and obtain

$$
\bar{P}_{1}\left(\tilde{Q}_{1}, \tilde{R}_{2}\right)=\left(\tilde{Q}_{1}-\pi_{1}^{H}\right) \Gamma_{0}\left(\bar{\rho}_{1}\left(\tilde{Q}_{1}, \tilde{R}_{2}\right)-\tilde{\rho}_{1}\left(\tilde{Q}_{1}, \tilde{Q}_{2}\right)\right) .
$$

As $\tilde{Q}_{1}$ has to be a weighted average of $\pi_{1}^{L}$ and $\pi_{1}^{H}$, the first term on the RHS of (A42), $\tilde{Q}_{1}-\pi_{1}^{H}$, is negative. Due to the Assumption (3.1), the second term on the RHS of (A42), $\bar{\rho}_{1}\left(\tilde{Q}_{1}, \tilde{R}_{2}\right)-\tilde{\rho}_{1}\left(\tilde{Q}_{1}, \tilde{Q}_{2}\right)$, is also negative, and it follows that $\bar{P}_{1}\left(\tilde{Q}_{1}, \tilde{R}_{2}\right)>0$.

In the same way, we determine the sign of $\bar{P}_{2}\left(\tilde{Q}_{1}, \tilde{R}_{2}\right)$ : Given that $\tilde{R}_{2}=\tilde{Q}_{1} \tilde{Q}_{2}$, each type's $i$ consumption level in the second retirement period is the same (see Proof of Lemma 4). Hence, $\tilde{A}_{2}^{i}\left(\tilde{Q}_{1}, \tilde{Q}_{2}\right)=\bar{D}_{2}^{i}\left(\tilde{Q}_{1}, \tilde{R}_{2}\right)$ for $i=L, H$, from which it is immediate that

$$
\tilde{\rho}_{2}\left(\tilde{Q}_{1}, \tilde{Q}_{2}\right)=\bar{\rho}_{2}\left(\tilde{Q}_{1}, \tilde{R}_{2}\right) .
$$

Using this, together with $\Gamma_{1}=\Gamma_{0} \pi_{1}^{H} / \pi_{1}^{L}$ (remember the definitions of $\Gamma_{t}, t=0,1$, and (3.4)), (3.5) can be transformed to

$$
\pi_{1}^{L} \pi_{2}^{L}+\pi_{1}^{H} \pi_{2}^{H} \Gamma_{0} \bar{\rho}_{2}\left(\tilde{Q}_{1}, \tilde{R}_{2}\right)=\tilde{Q}_{2}\left(\pi_{1}^{L}+\pi_{1}^{H} \Gamma_{0} \bar{\rho}_{2}\left(\tilde{Q}_{1}, \tilde{R}_{2}\right)\right) .
$$

Substituting this term, together with $\tilde{R}_{2}=\tilde{Q}_{1} \tilde{Q}_{2}$, into $\bar{P}_{2}\left(\tilde{Q}_{1}, \tilde{R}_{2}\right)$, we obtain

$$
\bar{P}_{2}\left(\tilde{Q}_{1}, \tilde{R}_{2}\right)=\tilde{Q}_{2}\left(\tilde{Q}_{1}-\pi_{1}^{L}+\left(\tilde{Q}_{1}-\pi_{1}^{H}\right) \Gamma_{0} \bar{\rho}_{2}\left(\tilde{Q}_{1}, \tilde{R}_{2}\right)\right)
$$


and further, by use of (3.3),

$$
\bar{P}_{2}\left(\tilde{Q}_{1}, \tilde{R}_{2}\right)=\tilde{Q}_{2}\left(\tilde{Q}_{1}-\pi_{1}^{H}\right) \Gamma_{0}\left(\bar{\rho}_{2}\left(\tilde{Q}_{1}, \tilde{R}_{2}\right)-\tilde{\rho}_{1}\left(\tilde{Q}_{1}, \tilde{Q}_{2}\right)\right)
$$

As $\tilde{Q}_{1}<\pi_{1}^{H}$ and $\bar{\rho}_{2}\left(\tilde{Q}_{1}, \tilde{R}_{2}\right)>\tilde{\rho}_{1}\left(\tilde{Q}_{1}, \tilde{Q}_{2}\right)$, as shown in Lemma 8 , the RHS of (A46) is negative, hence $\bar{P}_{2}\left(\tilde{Q}_{1}, \tilde{R}_{2}\right)<0$.

The assumptions $\partial \bar{\rho}_{1}\left(Q_{1}, R_{2}\right) / \partial R_{2}=0$ and $\partial \bar{\rho}_{2}\left(Q_{1}, R_{2}\right) / \partial Q_{1}=0$ imply that $\partial \bar{P}_{1}\left(Q_{1}, R_{2}\right) / \partial R_{2}=0$ and $\partial \bar{P}_{2}\left(Q_{1}, R_{2}\right) / \partial Q_{1}=0$. From this and (3.9), together with $\bar{P}_{1}\left(\tilde{Q}_{1}, \tilde{R}_{2}\right)>0$ and $\bar{P}_{2}\left(\tilde{Q}_{1}, \tilde{R}_{2}\right)<0$, resp., it follows for the zero-profit prices in the simultaneous regime that $\bar{Q}_{1}<\tilde{Q}_{1}$, and $\bar{R}_{2}>\tilde{R}_{2}\left(=\tilde{Q}_{1} \tilde{Q}_{2}\right)$, resp.

Proof of Lemma 10: By use of (2.8a) and (2.9), we obtain the condition for optimal consumption in both retirement periods $t=1,2$, in sequential regime as

$$
\frac{u^{\prime}\left(\tilde{c}_{1}^{i}\right)}{\pi_{2}^{i} u^{\prime}\left(\tilde{c}_{2}^{i}\right)}=\frac{1}{Q_{2}} \quad \text { for } i=L, H,
$$

and by use of (A21) the equivalent condition in the simultaneous regime as

$$
\frac{u^{\prime}\left(\bar{c}_{1}^{i}\right)}{\pi_{2}^{i} u^{\prime}\left(\bar{c}_{2}^{i}\right)}=\frac{Q_{1}}{R_{2}} \quad \text { for } i=L, H .
$$

As $\pi_{2}^{H}>\pi_{2}^{L}$, we find that $u^{\prime}\left(\tilde{c}_{1}^{H}\right) / u^{\prime}\left(\tilde{c}_{2}^{H}\right)>u^{\prime}\left(\tilde{c}_{1}^{L}\right) / u^{\prime}\left(\tilde{c}_{2}^{L}\right)$ must hold in order to satisfy (A47) and that $u^{\prime}\left(\bar{c}_{1}^{H}\right) / u^{\prime}\left(\bar{c}_{2}^{H}\right)>u^{\prime}\left(\bar{c}_{1}^{L}\right) / u^{\prime}\left(\bar{c}_{2}^{L}\right)$ must hold to satisfy (A48). From this, together with $u^{\prime \prime}\left(c_{t}^{i}\right)<0$, it follows that $\tilde{c}_{2}^{H} / \tilde{c}_{1}^{H}>\tilde{c}_{2}^{L} / \tilde{c}_{1}^{L}, \bar{c}_{2}^{H} / \bar{c}_{1}^{H}>\bar{c}_{2}^{L} / \bar{c}_{1}^{L}$, resp., is fulfilled irrespective of the optimal value of $c_{0}^{i}$, given homothetic preferences.

We know from Lemma 9 that $\bar{Q}_{1}<\tilde{Q}_{1}$ and $\tilde{Q}_{1} \tilde{Q}_{2}<\bar{R}_{2}$. Hence $1 / \tilde{Q}_{2}>$ $\bar{Q}_{1} / \bar{R}_{2}$, which-due to analogous considerations as above-imply that $\tilde{c}_{2}^{i} / \tilde{c}_{1}^{i}>\bar{c}_{2}^{i} / \bar{c}_{1}^{i}$ holds.

\section{Proof of Proposition 4:}

(i) We begin by showing that $\tilde{c}_{0}^{i}\left(Q_{1}, Q_{2}\right)=\bar{c}_{0}^{i}\left(Q_{1}, R_{2}\right), i=L, H$, at any prices $Q_{1}, Q_{2}$ and $R_{2}$ : Setting $\sigma=1$, and substituting the first equation in (A.36) and $D_{2}^{i}=0$ into (2.1) gives the same optimal consumption level $c_{0}^{i}=$ $w_{0} /\left(1+\pi_{1}^{i}+\pi_{1}^{i} \pi_{2}^{i}\right)$ in period 0 , as substituting both equations in (A37) into (2.1). From this, together with $\bar{Q}_{1} \tilde{Q}_{2}<\bar{R}_{2}$ (due to Lemma 9), it follows that the budget lines $\widetilde{B L}^{i}$ and $\overline{B L}^{i}$ in both regimes intersect and that the sequential regime allows larger consumption bundles $\left(c_{1}^{i}, c_{2}^{i}\right)$ to the left of the point of intersection, but smaller consumption bundles to the right.

(ii) Secondly, we calculate the slope of the indifference curve of each type $i=L, H$, going through the point of intersection of $\widetilde{B L}^{i}$ and $\overline{B L}^{i}$, to show then in step (iii) that at this point of intersection the slope of type's $L$ indifference curve is steeper than the slope of $\widetilde{B L}^{i}$ and that the slope of type's $H$ indifference curve is flatter than the slope of $\overline{B L}^{i}$. By use of $\tilde{c}_{0}^{i}=\bar{c}_{0}^{i}$, we solve (3.10) and (3.11) to obtain the 
point of intersection of budget lines $\widetilde{B L}^{i}$ and $\overline{B L}^{i}$ of an individual $i=L, H$, as

$$
c_{1}^{i}(S) \equiv \frac{\bar{R}_{2}-\tilde{Q}_{1} \tilde{Q}_{2}}{\tilde{Q}_{1}\left(\bar{R}_{2}-\bar{Q}_{1} \tilde{Q}_{2}\right)}\left(w_{0}-\bar{c}_{0}^{i}\right), \quad c_{2}^{i}(S) \equiv \frac{\tilde{Q}_{1}-\bar{Q}_{1}}{\tilde{Q}_{1}\left(\bar{R}_{2}-\bar{Q}_{1} \tilde{Q}_{2}\right)}\left(w_{0}-\bar{c}_{0}^{i}\right) .
$$

For logarithmic utility the slope of an indifference curve of type $i=L, H$, denoted by $\mathrm{MRS}_{1,2}^{i}$, is equal to $\operatorname{MRS}_{1,2}^{i} \equiv-c_{2}^{i} /\left(\pi_{2}^{i} c_{1}^{i}\right)$. Substituting (A49) into this term yields

$$
\operatorname{MRS}_{1,2}^{i}(S)=-\frac{\tilde{Q}_{1}-\bar{Q}_{1}}{\pi_{2}^{i}\left(\bar{R}_{2}-\tilde{Q}_{1} \tilde{Q}_{2}\right)}
$$

In order to show that $\operatorname{MRS}_{1,2}^{L}(S)<-1 / \tilde{Q}_{2}$ and that $\operatorname{MRS}_{1,2}^{H}(S)>-\bar{Q}_{1} / \bar{R}_{2}$, we have to reduce (A50) by use of zero-profit prices in either regime: As all demandratios $\tilde{\rho}_{t}, \bar{\rho}_{t}, t=1,2$, i.e. (use the respective demand in (A36) and (A37) for $\sigma=1)$

$$
\begin{array}{ll}
\bar{\rho}_{1}=\frac{\pi_{1}^{H}\left(1+\pi_{1}^{L}+\pi_{1}^{L} \pi_{2}^{L}\right)}{\pi_{1}^{L}\left(1+\pi_{1}^{H}+\pi_{1}^{H} \pi_{2}^{H}\right)}, & \bar{\rho}_{2}=\frac{\pi_{2}^{H}}{\pi_{2}^{L}} \bar{\rho}_{1}, \\
\tilde{\rho}_{1}=\frac{1+\pi_{2}^{H}}{1+\pi_{2}^{L}} \bar{\rho}_{1}, & \tilde{\rho}_{2}=\bar{\rho}_{2},
\end{array}
$$

are independent of prices, these can be computed explicitly from the respective zero-profit conditions (3.3), (3.5)-(3.7). By substituting from (3.3)

$$
\tilde{Q}_{1}=\frac{\pi_{1}^{L}+\pi_{1}^{H} \Gamma_{0} \tilde{\rho}_{1}}{1+\Gamma_{0} \tilde{\rho}_{1}}
$$

and from (3.6)

$$
\bar{Q}_{1}=\frac{\pi_{1}^{L}+\pi_{1}^{H} \Gamma_{0} \bar{\rho}_{1}}{1+\Gamma_{0} \bar{\rho}_{1}}
$$

into the numerator on the RHS in (A50), we derive

$$
\tilde{Q}_{1}-\bar{Q}_{1}=\frac{\left(\pi_{1}^{H}-\pi_{1}^{L}\right) \Gamma_{0}\left(\tilde{\rho}_{1}-\bar{\rho}_{1}\right)}{\left(1+\Gamma_{0} \tilde{\rho}_{1}\right)\left(1+\Gamma_{0} \bar{\rho}_{1}\right)}
$$

Substituting from (3.7)

$$
\bar{R}_{2}=\frac{\pi_{1}^{L} \pi_{2}^{L}+\pi_{1}^{H} \pi_{2}^{H} \Gamma_{0} \bar{\rho}_{2}}{1+\Gamma_{0} \bar{\rho}_{2}}
$$


and from (3.5), together with $\Gamma_{1}=\Gamma_{0} \pi_{1}^{H} / \pi_{1}^{L}$,

$$
\tilde{Q}_{2}=\frac{\pi_{1}^{L} \pi_{2}^{L}+\pi_{1}^{H} \pi_{2}^{H} \Gamma_{0} \tilde{\rho}_{2}}{\pi_{1}^{L}+\pi_{1}^{H} \Gamma_{0} \tilde{\rho}_{2}}
$$

and the second equation in (A52) into the price ratio $\bar{R}_{2} / \tilde{Q}_{2}$, we obtain

$$
\frac{\bar{R}_{2}}{\tilde{Q}_{2}}=\frac{\pi_{1}^{L}+\pi_{1}^{H} \Gamma_{0} \bar{\rho}_{2}}{1+\Gamma_{0} \bar{\rho}_{2}}
$$

which in turn is used, together with (A53), to write the denominator on the RHS in (A50) as

$$
\bar{R}_{2}-\tilde{Q}_{1} \tilde{Q}_{2}=\tilde{Q}_{2}\left(\frac{\pi_{1}^{L}+\pi_{1}^{H} \Gamma_{0} \bar{\rho}_{2}}{1+\Gamma_{0} \bar{\rho}_{2}}-\frac{\pi_{1}^{L}+\pi_{1}^{H} \Gamma_{0} \tilde{\rho}_{1}}{1+\Gamma_{0} \tilde{\rho}_{1}}\right)=\tilde{Q}_{2} \frac{\left(\pi_{1}^{H}-\pi_{1}^{L}\right) \Gamma_{0}\left(\bar{\rho}_{2}-\tilde{\rho}_{1}\right)}{\left(1+\Gamma_{0} \bar{\rho}_{2}\right)\left(1+\Gamma_{0} \tilde{\rho}_{1}\right)} .
$$

Substituting (A55) and (A59) into (A50) yields

$$
\operatorname{MRS}_{1,2}^{i}(S)=-\frac{\left(\tilde{\rho}_{1}-\bar{\rho}_{1}\right)\left(1+\Gamma_{0} \bar{\rho}_{2}\right)}{\pi_{2}^{i} \tilde{Q}_{2}\left(\bar{\rho}_{2}-\tilde{\rho}_{1}\right)\left(1+\Gamma_{0} \bar{\rho}_{1}\right)}
$$

Calculating the difference $\tilde{\rho}_{1}-\bar{\rho}_{1}$ by use of the first relation in (A52), as well as the difference $\bar{\rho}_{2}-\bar{\rho}_{1}$, by use of the second relation in (A51), i.e.

$$
\tilde{\rho}_{1}-\bar{\rho}_{1}=\frac{\pi_{2}^{H}-\pi_{2}^{L}}{1+\pi_{2}^{L}} \bar{\rho}_{1}, \quad \bar{\rho}_{2}-\bar{\rho}_{1}=\frac{\pi_{2}^{H}-\pi_{2}^{L}}{\pi_{2}^{L}\left(1+\pi_{2}^{L}\right)} \bar{\rho}_{1}
$$

and substituting these two equations into $(\mathrm{A} 60), \mathrm{MRS}_{1,2}^{i}(S)$ reduces further to

$$
\operatorname{MRS}_{1,2}^{i}(S)=-\frac{\pi_{2}^{L}\left(1+\Gamma_{0} \bar{\rho}_{2}\right)}{\pi_{2}^{i} \tilde{Q}_{2}\left(1+\Gamma_{0} \bar{\rho}_{1}\right)} .
$$

(iii) Now, we are ready to show that $\operatorname{MRS}_{1,2}^{L}(S)<-1 / \tilde{Q}_{2}$ and $\operatorname{MRS}_{1,2}^{H}(S)>$ $-\bar{Q}_{1} / \bar{R}_{2}$. Substituting the second equation in (A51) into (A62) for $i=L$ yields

$$
\operatorname{MRS}_{1,2}^{L}(S)=-\frac{\left(1+\Gamma_{0} \bar{\rho}_{1} \pi_{2}^{H} / \pi_{2}^{L}\right)}{\tilde{Q}_{2}\left(1+\Gamma_{0} \bar{\rho}_{1}\right)},
$$

which is smaller than $-1 / \tilde{Q}_{2}$, as $\pi_{2}^{H}>\pi_{2}^{L}$.

By substituting (A58) into (A62) for $i=H$ we get

$$
\operatorname{MRS}_{1,2}^{H}(S)=-\frac{\pi_{2}^{L}\left(\pi_{1}^{L}+\pi_{1}^{H} \Gamma_{0} \bar{\rho}_{2}\right)}{\pi_{2}^{H} \bar{R}_{2}\left(1+\Gamma_{0} \bar{\rho}_{1}\right)},
$$


which by use of the second equation in (A51) can be transformed to

$$
\operatorname{MRS}_{1,2}^{H}(S)=-\frac{\pi_{1}^{L} \pi_{2}^{L} / \pi_{2}^{H}+\pi_{1}^{H} \Gamma_{0} \bar{\rho}_{1}}{\bar{R}_{2}\left(1+\Gamma_{0} \bar{\rho}_{1}\right)} .
$$

By use of (A54) the slope of $\overline{B L}^{i}$ can be written as

$$
-\frac{\bar{Q}_{1}}{\bar{R}_{2}}=-\frac{\pi_{1}^{L}+\pi_{1}^{H} \Gamma_{0} \bar{\rho}_{1}}{\bar{R}_{2}\left(1+\Gamma_{0} \bar{\rho}_{1}\right)} .
$$

From (A64) and (A66) it is immediate that $\operatorname{MRS}_{1,2}^{H}(S)>-\bar{Q}_{1} / \bar{R}_{2}$ due to $\pi_{2}^{L}<\pi_{2}^{H}$.

(iv) Finally, the inequality $\operatorname{MRS}_{1,2}^{L}(S)<-1 / \tilde{Q}_{2}$ implies that the optimal consumption bundle $\left(\tilde{c}_{1}^{L}, \tilde{c}_{2}^{L}\right)$ of type $L$ in sequential regime lies to the right of the point of intersection of $\widetilde{B L}^{L}$ and $\overline{B L}^{L}$. Moreover, we know from part (ii) of Lemma 10 that $\tilde{c}_{2}^{L} / \tilde{c}_{1}^{L}>\bar{c}_{2}^{L} / \bar{c}_{1}^{L}$, thus her optimal bundle $\left(\bar{c}_{1}^{L}, \bar{c}_{2}^{L}\right)$ in the simultaneous regime also lies to the right of the intersection of $\widetilde{B L}^{L}$ and $\overline{B L}^{L}$. Consequently, since the simultaneous regime allows higher consumption possibilities to the right of the point of intersection, it is preferred by a type- $L$ individual. A similar argument shows that, due to $\operatorname{MRS}_{1,2}^{H}(S)>-\bar{Q}_{1} / \bar{R}_{2}$ and $\tilde{c}_{2}^{H} / \tilde{c}_{1}^{H}>\bar{c}_{2}^{H} / \bar{c}_{1}^{H}$, the optimal consumption bundles of type $H$ lie, in both regimes, to the left of the point of intersection of $\widetilde{B L}^{H}$ and $\overline{B L}^{H}$. Thus, the sequential regime is preferred by the type- $H$ individuals.

\section{References}

ABEL, A.B. [1986]: "Capital Accumulation and Uncertain Lifetime with Adverse Selection,” Econometrica, 54, 1079-1097.

BOLTON, P. and DEWATRIPONT, M. [2005]: Contract Theory, Cambridge: MIT Press.

BRUGIAVINI, A. [1993]: "Uncertain Resolution and the Timing of Annuity Purchases," Journal of Public Economics, 31-62.

BRUNNER, J.K. and PECH, S. [2005]: “Adverse Selection in the Annuity Market when Payoffs Vary Over the Time of Retirement," Journal of Institutional and Theoretical Economics, 161, 155-183.

ECKSTEIN, Z., EICHENBAUM, M., and PELED, D. [1985]: "Uncertain Lifetimes and the Welfare Enhancing Properties of Annuity Markets and Social Security," Journal of Public Economics, 26, 303-326.

FINKELSTEIN, A. and POTERBA, J.M. [2002]: "Selection Effects in the United Kingdom Individual Annuities Market,” Economic Journal, 112(476), 28-50.

FRIEDMAN, B.M. and WARSHAWSKY, M.J. [1990]: "The Cost of Annuities: Implications for Saving Behaviour and Bequests," Quaterly Journal of Economics, 105, 135-154.

MITCHELL, O.S., POTERBA, J.M., WARSHAWSKY, M.J., and BROWN, J.R. [1999]: "New Evidence on the Money's Worth of Individual Annuities," American Economic Review, 89, 1299-1318.

PAULY, M.V. [1974]: "Overinsurance and Public Provision of Insurance: The Roles of Moral Hazard and Adverse Selection,” Quarterly Journal of Economics, 88, 44-62.

PECH, S. [2004]: "Tax Incentives for Private Life Annuities and the Social Security Reform: Effects on Consumption and on Adverse Selection," FinanzArchiv, 60(4), 556-592.

POTERBA, J.M. [1997]: “The History of Annuities in the United States,” NBER Working Paper 6001.

ROTHSCHILD, M. and STIGLITZ, J. [1976]: "Equilibrium in Competitive Insurance Markets: An Essay on the Economics of Imperfect Information," Quarterly Journal of Economics, 90, 629-649. 
TOWNLEY, P.G.C. and BOADWAY, R.W. [1988]: "Social Security and the Failure of Annuity Markets," Journal of Public Economics, 35, 75-96.

WALLISER, J. [2000]: "Adverse Selection in the Annuities Market and the Impact of Privatizing Social Security," Scandinavian Journal of Economics, 102, 373-393.

WILSON, Ch. [1977]: “A Model of Insurance Markets with Incomplete Information,” Journal of Economic Theory, 16, 167-207

YAARI, M.E. [1965]: "Uncertain Lifetime, Life Insurance, and the Theory of the Consumer," Review of Economic Studies, 32, 137-150.

YAGI, T. and NISHIGAKI, Y. [1993]: "The Inefficiency of Private Constant Annuities," Journal of Risk and Insurance, 60, 385-412. 